\title{
ABSTRACT STOCHASTIC INTEGRODIFFERENTIAL DELAY EQUATIONS
}

\author{
DAVID N. KECK AND MARK A. MCKIBBEN
}

Received 26 August 2004 and in revised form 2 November 2004

We investigate a class of abstract stochastic integrodifferential delay equations dependent upon a family of probability measures in a separable Hilbert space. We establish the existence and uniqueness of a mild solution, along with various continuous dependence estimates and Markov (weak and strong) properties of this solution. This is followed by a convergence result concerning the strong solutions of the Yosida approximations of our equation, from which we deduce the weak convergence of the measures induced by these strong solutions to the measure induced by the mild solution of the primary problem under investigation. Next, we establish the $p$ th moment and almost sure exponential stability of the mild solution. Finally, an analysis of two examples, namely a generalized stochastic heat equation and a Sobolev-type evolution equation, is provided to illustrate the applicability of the general theory.

\section{Introduction}

In this paper, we will initiate an investigation of a class of abstract delay integrodifferential stochastic evolution equations of the general form

$$
\begin{aligned}
x^{\prime}(t)+A x(t)= & f_{1}\left(t, x_{t}, \mu(t)\right)+\int_{0}^{t} K_{1}(t, s) f_{2}\left(s, x_{s}, \mu(s)\right) d s \\
& +\int_{0}^{t} K_{2}(t, s) f_{3}\left(s, x_{s}, \mu(s)\right) d W(s), \quad 0 \leq t \leq T, \\
x(t)= & \phi(t), \quad-r \leq t \leq 0,
\end{aligned}
$$

in a real separable Hilbert space $H$. Here, $W$ is a given $K$-valued Wiener process associated with a positive, nuclear covariance operator $Q ; A$ is a linear (possibly unbounded) operator which generates a strongly continuous semigroup $\{S(t): t \geq 0\}$ on $H ; K_{1}(t, s)$ and $K_{2}(t, s)$ are bounded, linear operators on $H ; f_{i}:[0, T] \times C_{r} \times \wp_{\lambda^{2}}(H) \rightarrow H(i=1,2)$ and $f_{3}:[0, T] \times C_{r} \times \wp_{\lambda^{2}}(H) \rightarrow B L(K ; H)$ (where $K$ is a real separable Hilbert space, $B L(K ; H)$ denotes the space of all bounded, linear operators from $K$ into $H$, and $\wp_{\lambda^{2}}(H)$ denotes a particular subset of probability measures on $H$ ) are given mappings; $\mu(t)$ is 
the probability law of $x(t)$ (i.e., $\mu(t)(A)=P(\{\omega \in \Omega: x(t, \omega) \in A\})$, for each $A \in \mathscr{B}(H))$; and $\phi \in L^{2}\left(\Omega ; C_{r}\right)$ is an $\mathscr{F}_{0}$-measurable random variable independent of $W$ with almost surely continuous paths. (See Section 2 for notation and spaces.) The second integral in (1.1) is taken in the sense of Itó. A complete discussion of the construction of the Itó integral can be found in [10].

Stochastic partial functional differential equations with finite delay arise naturally in the mathematical modeling of phenomena in the natural sciences (see $[22,30,34])$ and have begun to receive a significant amount of attention. Often, a more accurate model of such phenomena can be formulated by allowing the nonlinear perturbations to depend on the probability law of the state process at time $t$. A prototypical example in the finite-dimensional setting would be an interacting $N$-particle system in which (1.1) describes the dynamics of the particles $x_{1}, \ldots, x_{N}$ moving in the space $H$ in which the probability measure $\mu$ is taken to be the empirical measure $\mu_{N}(t)=(1 / N) \sum_{k=1}^{N} \delta_{x_{k}(t)}$, where $\delta_{x_{k}(t)}$ denotes the Dirac measure. Researchers have investigated related models concerning diffusion processes in the finite-dimensional case (e.g., see $[11,12,24])$. The infinitedimensional version of such models in a Hilbert space setting has only recently been examined (see $[1,19])$. The motivation of the present work lies primarily in formulating an extension of the work in $[1,7,13,18]$ to a more general class of abstract integrodifferential stochastic evolution equations with finite delay. Since dynamical systems with memory can lead to such random integrodifferential equations (cf. $[5,9,17,23])$, the present investigation is warranted.

The following is the outline of the paper. First, we make precise the necessary notation and function spaces, and gather certain preliminary results in Section 2. We then formulate the main results concerning the existence and uniqueness of mild solutions to (1.1), along with their regularity and stochastic properties in Section 3. Next, considering the Yosida approximations of (1.1), two convergence results are established in Section 4, while Section 5 is devoted to a discussion of certain asymptotic behavior results for (1.1). Finally, we conclude the paper with a discussion of two concrete examples (a generalized stochastic heat equation and a Sobolev-type stochastic equation) in Section 6.

\section{Preliminaries}

For details of this section, we refer the reader to $[4,6,10,14,25,26,29,34]$ and the references therein. Throughout this paper, $H$ and $K$ will denote real separable Hilbert spaces with respective norms $\|\cdot\|$ and $\|\cdot\|_{K}$. Let $(\Omega, \mathscr{F}, P)$ be a complete probability space equipped with a normal filtration $\left\{\mathscr{F}_{t}\right\}_{t \geq 0}$ (i.e., a right-continuous, increasing family of sub $\sigma$-algebras of $\mathscr{F}$ ). For $t<0, \mathscr{F}_{t}$ is taken to be $\mathscr{F}_{0}$. For brevity, we suppress the dependence of all mappings on $\omega$ throughout the manuscript.

Definition 2.1. A stochastic process $\{W(t): t \geq 0\}$ in a real separable Hilbert space $H$ is a Wiener process if for each $t \geq 0$,

(i) $W(t)$ has continuous sample paths and independent increments,

(ii) $W(t) \in L^{2}(\Omega ; H)$ and $E(W(t))=0$,

(iii) $\operatorname{Cov}(W(t)-W(s))=(t-s) Q$, where $Q \in B L(K ; H)$ is a nonnegative nuclear operator. 
The following spaces of measures coincide with those used in [1]; we recall them here for convenience. First, $\mathscr{S}(H)$ stands for the Borel class on $H$ and $\wp(H)$ represents the space of all probability measures defined on $\mathscr{B}(H)$ equipped with the weak convergence topology. Let $\lambda(x)=1+\|x\|, x \in H$. Define the space

$$
\begin{aligned}
\mathscr{C}(H)=\{\varphi: H \rightarrow H \mid \varphi \text { is continuous and } \\
\left.\|\varphi\|_{\mathscr{C}}=\sup _{x \in H} \frac{\|\varphi(x)\|}{\lambda^{2}(x)}+\sup _{x \neq y \text { in } H} \frac{\|\varphi(x)-\varphi(y)\|}{\|x-y\|}<\infty\right\},
\end{aligned}
$$

and for $p \geq 1$, let

$$
\begin{gathered}
\wp_{\lambda^{p}}^{s}(H)=\{m: H \rightarrow \mathbb{R} \mid m \text { is a signed measure on } H \text { such that } \\
\left.\|m\|_{\lambda^{p}}=\int_{H} \lambda^{p}(x)|m|(d x)<\infty\right\}
\end{gathered}
$$

where $|m|=m^{+}+m^{-}, m=m^{+}-m^{-}$is the Jordan decomposition of $m$. Then, we can define the space $\wp_{\lambda^{2}}(H)=\wp_{\lambda^{2}}^{s}(H) \cap \wp(H)$ equipped with the metric $\rho$ given by

$$
\rho\left(\nu_{1}, v_{2}\right)=\sup \left\{\int_{H} \varphi(x)\left(\nu_{1}-v_{2}\right)(d x):\|\varphi\|_{\mathscr{C}} \leq 1\right\}
$$

It is known that $\left(\wp_{\lambda^{2}}(H), \rho\right)$ is a complete metric space. The space of all continuous $\wp_{\lambda^{2}}(H)$-valued functions defined on $[-r, T]$, denoted by $\mathscr{C}_{\lambda^{2}}=\mathscr{C}_{\lambda^{2}}\left([-r, T] ;\left(\wp_{\lambda^{2}}(H), \rho\right)\right)$, is complete when equipped with the metric

$$
D_{T}\left(\nu_{1}, \nu_{2}\right)=\sup _{t \in[-r, T]} \rho\left(\nu_{1}(t), \nu_{2}(t)\right) \quad \forall \nu_{1}, \nu_{2} \in \mathscr{C}_{\lambda^{2}} .
$$

Next, let $r>0$. We can associate to any continuous, $\mathscr{F}_{t}$-adapted, $H$-valued stochastic process $z(t): \Omega \rightarrow H$ another $C_{r}$-valued stochastic process $z_{t}: \Omega \rightarrow C_{r}$ by setting $z_{t}(s)=$ $z(t+s)$, for all $t \geq 0$ and $-r \leq s \leq 0$, where we denote by $C_{r} \equiv C([-r, 0] ; H)$ the space of all continuous functions from $[-r, 0]$ into $H$, equipped with the sup norm given by

$$
\|z\|_{C_{r}}=\left(\sup _{-r \leq \theta \leq 0}\left\|z_{t}(\theta)\right\|^{2}\right)^{1 / 2} .
$$

Subsequently, we can define the space

$$
X_{T, 2}=\left\{z \in C\left([-r, T] ; L^{2}(\Omega ; H)\right) \mid z \text { is } \mathscr{F}_{t} \text {-adapted and }\|z\|_{X_{T, 2}}<\infty\right\},
$$

which is a Banach space when equipped with the norm

$$
\|z\|_{X_{T, 2}}=\sup _{0 \leq t \leq T}\left(E\left\|z_{t}\right\|_{C_{r}}^{2}\right)^{1 / 2} .
$$


Proposition 1.9 in [16], and variations thereof (as needed for the delay case), are used throughout the manuscript. We recall it here for convenience.

Lemma 2.2. Let $G:[0, T] \times \Omega \rightarrow B L(K, H)$ be strongly measurable with $\int_{0}^{T} E\|G(t)\|^{p} d t<$ $\infty$. Then,

$$
E\left\|\int_{0}^{t} G(s) d W(s)\right\|^{p} \leq L_{G} \int_{0}^{t} E\|G(s)\|_{B L(K, H)}^{p} d s
$$

where $L_{G}=[(1 / 2) p(p-1)]^{p / 2}(\operatorname{Tr} Q)^{p / 2} T^{(p / 2)-1}$.

In addition to the familiar Young, Hölder, and Minkowski inequalities, the inequality of the form $\left(\sum_{i=1}^{n} a_{i}\right)^{m} \leq m^{n-1} \sum_{i=1}^{n} a_{i}^{m}$, where $a_{i}$ is a nonnegative constant $(i=1, \ldots, n)$ and $m, n \in \mathbb{N}$, will be used to establish various estimates. Finally, the following integral inequality [25, page 38] plays an important role in the proofs of certain results.

Lemma 2.3. Let $u, \psi_{1}, \psi_{2}$, and $\psi_{3}$ be nonnegative continuous functions defined on $\mathbb{R}^{+}$and $u_{0}$ a nonnegative constant. If

$$
\begin{aligned}
u(t) \leq & u_{0}+\int_{0}^{t} \psi_{1}(s) u(s) d s+\int_{0}^{t} \psi_{1}(s)\left(\int_{0}^{s} \psi_{2}(\tau) u(\tau) d \tau\right) d s \\
& +\int_{0}^{t} \psi_{1}(s)\left(\int_{0}^{s} \psi_{2}(\tau)\left(\int_{0}^{\tau} \psi_{3}(\theta) u(\theta) d \theta\right) d \tau\right) d s
\end{aligned}
$$

for all $t \in \mathbb{R}^{+}$, then

$$
\begin{aligned}
u(t) \leq u_{0}\left[1+\int_{0}^{t}\{\right. & \psi_{1}(s) \exp \left(\int_{0}^{s} \psi_{1}(\tau) d \tau\right) \\
& \left.\left.\times\left(1+\int_{0}^{s} \psi_{2}(\tau) \exp \left(\int_{0}^{\tau}\left[\psi_{2}(\theta)+\psi_{3}(\theta)\right] d \theta\right) d \tau\right)\right\} d s\right]
\end{aligned}
$$

for all $t \in \mathbb{R}^{+}$.

We conclude this section with some comments regarding probability measures. The probability measure $P$ induced by an $H$-valued random variable $X$, denoted $P_{X}$, is defined by $P \circ X^{-1}: \mathscr{B}(H) \rightarrow[0,1]$. A sequence $\left\{P_{n}\right\} \subset \wp(H)$ is said to be weakly convergent to $P$ if $\int_{\Omega} f d P_{n} \rightarrow \int_{\Omega} f d P$, for every bounded, continuous function $f: H \rightarrow \mathbb{R}$; in such case, we write $P_{n} \stackrel{w}{\rightarrow} P$. Next, a family $\left\{P_{n}\right\}$ is tight if for each $\varepsilon>0$, there exists a compact set $K_{\varepsilon}$ such that $P_{n}\left(K_{\varepsilon}\right) \geq 1-\varepsilon$, for all $n$. Prokhorov (see [4]) established the equivalence of tightness and relative compactness of a family of probability measures. Consequently, the Arzelá-Ascoli theorem can be used to establish tightness.

Definition 2.4. Let $P \in \wp(H)$ and $0 \leq t_{1}<t_{2}<\cdots<t_{k} \leq T$. Define $\pi_{t_{1}, \ldots, t_{k}}: C([0, T] ; H) \rightarrow$ $H^{k}$ by $\pi_{t_{1}, \ldots, t_{k}}(X)=\left(X\left(t_{1}\right), \ldots, X\left(t_{k}\right)\right)$. The probability measures induced by $\pi_{t_{1}, \ldots, t_{k}}$ are the finite-dimensional joint distributions of $P$.

Proposition 2.5 (see [21, page 37]). If a sequence $\left\{X_{n}\right\}$ of $H$-valued random variables converges weakly to an $H$-valued random variable $X$ in $L^{2}(\Omega ; H)$, then the sequence of 
finite-dimensional joint distributions corresponding to $\left\{P_{X_{n}}\right\}$ converges weakly to the finitedimensional joint distribution of $P_{X}$.

The next theorem, in conjunction with Proposition 2.5, is the main tool in establishing a convergence result in Section 4.

Theorem 2.6. Let $\left\{P_{n}\right\} \subset \wp(H)$. If the sequence of finite-dimensional joint distributions corresponding to $\left\{P_{n}\right\}$ converges weakly to the finite-dimensional joint distribution of $P$ and $\left\{P_{n}\right\}$ is relatively compact, then $P_{n} \stackrel{w}{\rightarrow} P$.

\section{Existence, uniqueness, regularity, and Markov properties}

We begin by looking at the existence and uniqueness of mild solutions to (1.1). We impose the following conditions on (1.1), which are assumed throughout the manuscript unless otherwise specified:

(A1) $A$ is the infinitesimal generator of a $C_{0}$-semigroup $\{S(t): t \geq 0\}$ on $H$ such that $\|S(t)\| \leq M \exp (\alpha t)$, for all $0 \leq t \leq T$, for some $M \geq 1$ and $\alpha>0$,

(A2) $f_{i}:[0, T] \times C_{r} \times \wp_{\lambda^{2}}(H) \rightarrow H(i=1,2)$ satisfies

(i) $\left\|f_{i}\left(t, x_{t}, \mu(t)\right)\right\| \leq \bar{M}_{f_{i}}\left[1+\left\|x_{t}\right\|_{C_{r}}+\|\mu(t)\|_{\lambda^{2}}\right]$,

(ii) $\left\|f_{i}\left(t, x_{t}, \mu(t)\right)-f_{i}\left(t, y_{t}, \nu(t)\right)\right\| \leq M_{f_{i}}\left[\left\|x_{t}-y_{t}\right\|_{C_{r}}+\rho(\mu(t), v(t))\right]$, globally on $[0, T] \times C_{r} \times \wp_{\lambda^{2}}(H)$, for some positive constants $M_{f_{i}}$ and $\bar{M}_{f_{i}}$,

(A3) $f_{3}:[0, T] \times C_{r} \times \wp_{\lambda^{2}}(H) \rightarrow B L(K, H)$ satisfies

(i) $\left\|f_{3}\left(t, x_{t}, \mu(t)\right)\right\|_{B L(K, H)} \leq \bar{M}_{f_{3}}\left[1+\left\|x_{t}\right\|_{C_{r}}+\|\mu(t)\|_{\lambda^{2}}\right]$,

(ii) $\left\|f_{3}\left(t, x_{t}, \mu(t)\right)-f_{3}\left(t, y_{t}, \nu(t)\right)\right\|_{B L(K, H)} \leq M_{f_{3}}\left[\left\|x_{t}-y_{t}\right\|_{C_{r}}+\rho(\mu(t), v(t))\right]$,

globally on $[0, T] \times C_{r} \times \wp_{\lambda^{2}}(H)$, for some positive constants $M_{f_{3}}$ and $\bar{M}_{f_{3}}$,

(A4) $\left\{K_{1}(t, s):(t, s) \in \Delta\right\} \cup\left\{K_{2}(t, s):(t, s) \in \Delta\right\} \subset B L(H, H)$ are such that $\left\|K_{1}(t, s)\right\|_{B L(H, H)} \leq M_{K_{1}}$ and $\left\|K_{2}(t, s)\right\|_{B L(H, H)} \leq M_{K_{2}}$, for all $(t, s) \in \Delta$, for some positive constants $M_{K_{1}}$ and $M_{K_{2}}$.

(Henceforth, we write $M_{S}=\max _{0 \leq t \leq T}\|S(t)\|$, which is finite by (A1).) A mild solution to (1.1) is defined as follows.

Definition 3.1. A continuous stochastic process $x:[-r, T] \rightarrow H$ is a mild solution of (1.1) if

(i) $x(t)$ is $\mathscr{F}_{t}$-adapted, for each $-r \leq t \leq T$,

(ii) $\int_{0}^{T}\|x(s)\|^{2} d s<\infty$, almost surely $[P]$,

(iii)

$$
\begin{aligned}
x(t)= & S(t) \phi(0) \\
& +\int_{0}^{t} S(t-s) f_{1}\left(s, x_{s}, \mu(s)\right) d s \\
& +\int_{0}^{t} S(t-s) \int_{0}^{s} K_{1}(s, \tau) f_{2}\left(\tau, x_{\tau}, \mu(\tau)\right) d \tau d s \\
& +\int_{0}^{t} S(t-s) \int_{0}^{s} K_{2}(s, \tau) f_{3}\left(\tau, x_{\tau}, \mu(\tau)\right) d W(\tau) d s \quad \forall 0 \leq t \leq T, \text { almost surely }[P],
\end{aligned}
$$

(iv) $x(t)=\phi(t),-r \leq t \leq 0$, almost surely $[P]$. 
The first result is as follows.

Theorem 3.2. Let $\phi \in L^{2}\left(\Omega ; C_{r}\right)$ be an $\mathscr{F}_{0}$-measurable random variable independent of $W$ with almost surely continuous paths. If $(A 1)-(A 4)$ hold, then (1.1) has a unique mild solution $x \in X_{T, 2}$ with corresponding probability law $\mu \in \mathscr{C}_{\lambda^{2}}$, provided that $\alpha_{1}+\alpha_{2}<1$, where $\alpha_{1}$ and $\alpha_{2}$ are positive constants independent of $T$ (cf. (3.13)).

Proof. Let $\mu \in \mathscr{C}_{\lambda^{2}}$ be fixed and define the solution map $\Phi: X_{T, 2} \rightarrow X_{T, 2}$ by

$$
\begin{aligned}
(\Phi x)(t)= & S(t) \phi(0)+\int_{0}^{t} S(t-s) f_{1}\left(s, x_{s}, \mu(s)\right) d s \\
& +\int_{0}^{t} S(t-s) \int_{0}^{s} K_{1}(s, \tau) f_{2}\left(\tau, x_{\tau}, \mu(\tau)\right) d \tau d s \\
& +\int_{0}^{t} S(t-s) \int_{0}^{s} K_{2}(s, \tau) f_{3}\left(\tau, x_{\tau}, \mu(\tau)\right) d W(\tau) d s \\
= & S(t) \phi(0)+\sum_{i=1}^{3} I_{i}^{x}(t), \quad 0 \leq t \leq T, \\
(\Phi x)(t)= & \phi(t), \quad-r \leq t \leq 0 .
\end{aligned}
$$

To see that $\Phi$ is well defined, we first verify the $L^{2}$-continuity of $\Phi$ on $[0, T]$. Let $x \in X_{T, 2}$, $0<t_{1}<T$, and $|h|$ be sufficiently small (so that all terms are well defined). Observe that

$$
\begin{aligned}
& E\left\|(\Phi x)\left(t_{1}+h\right)-(\Phi x)\left(t_{1}\right)\right\|^{2} \\
& \quad \leq 8\left[E\left\|\left(\left(S\left(t_{1}+h\right)-S\left(t_{1}\right)\right) \phi(0)\right)\right\|^{2}+\sum_{i=1}^{3} E\left\|I_{i}^{x}\left(t_{1}+h\right)-I_{i}^{x}\left(t_{1}\right)\right\|^{2}\right] .
\end{aligned}
$$

Since the semigroup property enables us to write

$$
E\left\|\left(\left(S\left(t_{1}+h\right)-S\left(t_{1}\right)\right) \phi(0)\right)\right\|^{2}=E\left\|\left(S(h)\left(S\left(t_{1}\right) \phi(0)\right)-S\left(t_{1}\right) \phi(0)\right)\right\|^{2},
$$

the strong continuity of $S(t)$ implies that the right-hand side of (3.4) goes to 0 as $|h| \rightarrow 0$. Next, using the Hölder inequality along with (A2) yields

$$
\begin{aligned}
& E\left\|\int_{t_{1}}^{t_{1}+h} S\left(t_{1}+h-s\right) f_{1}\left(s, x_{s}, \mu(s)\right) d s\right\|^{2} \\
& \quad \leq 4\left(\bar{M}_{f_{1}}\right)^{2} M_{S}^{2} h^{2}\left[1+\|x\|_{X_{T, 2}}^{2}+\sup _{t_{1} \leq s \leq t_{1}+h}\|\mu(s)\|_{\lambda^{2}}^{2}\right],
\end{aligned}
$$

which clearly goes to 0 as $|h| \rightarrow 0$. Also,

$$
\begin{aligned}
& E\left\|\int_{0}^{t_{1}}[S(h)-I] S\left(t_{1}-s\right) f_{1}\left(s, x_{s}, \mu(s)\right) d s\right\|^{2} \\
& \quad \leq T \int_{0}^{t_{1}}\left\|[S(h)-I] S\left(t_{1}-s\right)\right\|^{2} E\left\|f_{1}\left(s, x_{s}, \mu(s)\right)\right\|^{2} d s
\end{aligned}
$$


and subsequently, using $\left\|f_{1}\left(s, x_{s}, \mu(s)\right)\right\|^{2} \leq\left[1+\|x\|_{X_{T, 2}}^{2}+\sup _{0 \leq s \leq T}\|\mu(s)\|_{\lambda^{2}}^{2}\right]<\infty$, together with the strong continuity of $S(t)$, we can invoke the dominated convergence theorem to conclude that the right-hand side of (3.6) goes to 0 as $|h| \rightarrow 0$. Consequently, since $E\left\|I_{1}^{x}\left(t_{1}+h\right)-I_{1}^{x}\left(t_{1}\right)\right\|^{2}$ is dominated by a sum of constant multiples of the right-hand sides of (3.5) and (3.6), we conclude that it goes to 0 as $|h| \rightarrow 0$.

Next, an application of the Hölder inequality, together with Itó's formula in conjunction with Lemma 2.2, yields

$$
\begin{aligned}
& E\left\|I_{3}^{x}\left(t_{1}+h\right)-I_{3}^{x}\left(t_{1}\right)\right\|^{2} \\
& \leq 16 M_{K_{2}}^{2}\left(\bar{M}_{f_{3}}\right)^{2} L_{f_{3}}^{2} T\left[M_{S}^{2} \int_{t_{1}}^{t_{1}+h} \int_{0}^{s}\left[1+E\left\|x_{\tau}\right\|_{C_{r}}^{2}+\|\mu(\tau)\|_{\lambda^{2}}^{2}\right] d \tau d s\right. \\
& +\int_{0}^{t_{1}}\left\|[S(h)-I] S\left(t_{1}-s\right)\right\|^{2} \\
& \left.\quad \times\left(\int_{0}^{s}\left[1+E\left\|x_{\tau}\right\|_{C_{r}}^{2}+\|\mu(\tau)\|_{\lambda^{2}}^{2}\right] d \tau\right) d s\right],
\end{aligned}
$$

where $L_{f_{3}}$ is the constant of Lemma 2.2. Thus, the above reasoning can be used to conclude that the right-hand side of (3.7) goes to 0 as $|h| \rightarrow 0$. Similar computations can be used to show that the same is true of $E\left\|I_{2}^{x}\left(t_{1}+h\right)-I_{2}^{x}\left(t_{1}\right)\right\|^{2}$. Consequently, we can conclude (from (3.3)) that $\Phi$ is indeed $L^{2}$-continuous on $[0, T]$.

Next, to see that $\Phi\left(X_{T, 2}\right) \subset X_{T, 2}$, let $x \in X_{T, 2}$ and $t \in[0, T]$. Since $\phi \in L^{2}\left(\Omega ; C_{r}\right)$, it follows that

$$
\sup _{-r \leq \theta \leq 0}\left\{E\|(\Phi x)(t+\theta)\|^{2}:-r \leq t+\theta \leq 0\right\}<\infty .
$$

For all $-r \leq \theta \leq 0$ for which $t+\theta>0$, standard computations involving the Hölder inequality, (A2)-(A4), and Lemma 2.3 yield the following estimates:

$$
\begin{gathered}
E\left(\sup _{-r \leq \theta \leq 0}\|S(t+\theta) \phi(0)\|^{2}\right) \leq M_{S}^{2}\|\phi\|_{C_{r}}^{2}, \\
E\left(\sup _{-r \leq \theta \leq 0}\left\|I_{1}^{x}(t+\theta)\right\|^{2}\right) \leq 4\left(T M_{S} M_{f_{1}}\right)^{2}\left[1+\|x\|_{X_{T, 2}}^{2}+\sup _{0 \leq \theta \leq T}\|\mu(\theta)\|_{\lambda^{2}}^{2}\right], \\
E\left(\sup _{-r \leq \theta \leq 0}\left\|I_{2}^{x}(t+\theta)\right\|^{2}\right) \leq 4\left(T M_{S} M_{f_{2}} M_{K_{1}}\right)^{2}\left[1+\|x\|_{X_{T, 2}}^{2}+\sup _{0 \leq \theta \leq T}\|\mu(\theta)\|_{\lambda^{2}}^{2}\right], \\
E\left(\sup _{-r \leq \theta \leq 0}\left\|I_{3}^{x}(t+\theta)\right\|^{2}\right) \leq 4 T\left(M_{S} M_{f_{3}} M_{K_{2}} L_{f_{3}}\right)^{2}\left[1+\|x\|_{X_{T, 2}}^{2}+\sup _{0 \leq \theta \leq T}\|\mu(\theta)\|_{\lambda^{2}}^{2}\right] .
\end{gathered}
$$

Hence, we conclude that (cf. (3.2))

$$
\sup _{-r \leq \theta \leq 0}\left\{E\|(\Phi x)(t+\theta)\|^{2}: 0 \leq t+\theta \leq T\right\}<\infty .
$$


Thus, (3.8) and (3.10) together imply that $E\left\|(\Phi x)_{t}\right\|_{C_{r}}^{2}<\infty$, for all $t \in[0, T]$, so that $\Phi(x) \in X_{T, 2}$. Since the $\mathscr{F}_{t}$-measurability of $(\Phi x)(t)$ is easily verified, we conclude that $\Phi$ is well defined.

Next, we prove that $\Phi$ has a unique fixed point. Indeed, for any $x, y \in X_{T, 2}$, (3.2) implies

$$
E\left\|(\Phi x)_{t}-(\Phi y)_{t}\right\|_{C_{r}}^{2} \leq 4 E\left[\sup _{-r \leq \theta \leq 0}\left(\sum_{i=1}^{3}\left\|I_{i}^{x}(t+\theta)-I_{i}^{y}(t+\theta)\right\|^{2}\right)\right], \quad 0 \leq t \leq T .
$$

Standard computations yield

$$
\begin{aligned}
E\left\|(\Phi x)_{t}-(\Phi y)_{t}\right\|_{C_{r}}^{2} \leq 4 T M_{S}^{2}[ & M_{f_{1}}^{2} \int_{0}^{t} E\left\|x_{\theta}-y_{\theta}\right\|_{C_{r}}^{2} d \theta+T\left(T M_{f_{2}}^{2} M_{K_{1}}^{2}+M_{f_{3}}^{2} M_{K_{2}}^{2} L_{f_{3}}^{2}\right) \\
& \left.\times \int_{0}^{t} \int_{0}^{\theta} E\left\|x_{\tau}-y_{\tau}\right\|_{C_{r}}^{2} d \tau d \theta\right], \quad 0 \leq t \leq T .
\end{aligned}
$$

We proceed in two cases to prove that $\Phi^{N}$ is a strict contraction, for some $N$. First, if $T \leq 1$, then $T^{2} \leq 1$, so that we can continue (3.12) to obtain

$$
\begin{aligned}
& E\left\|(\Phi x)_{t}-(\Phi y)_{t}\right\|_{C_{r}}^{2} \\
& \quad \leq \alpha_{1} \int_{0}^{t} E\left\|x_{\theta}-y_{\theta}\right\|_{C_{r}}^{2} d \theta+\alpha_{2} \int_{0}^{t} \int_{0}^{\theta} E\left\|x_{\tau}-y_{\tau}\right\|_{C_{r}}^{2} d \tau d \theta, \quad 0 \leq t \leq T,
\end{aligned}
$$

where $\alpha_{1}=4 M_{S}^{2} M_{f_{1}}^{2}$ and $\alpha_{2}=4 M_{S}^{2}\left(M_{f_{2}}^{2} M_{K_{1}}^{2}+M_{f_{3}}^{2} M_{K_{2}}^{2} L_{f_{3}}^{2}\right)$ are independent of $T$. Inductively, it can be shown that for each $n \geq 1$, that if $\alpha_{n, j-n+1}$ is the $(j-n+1)$ th term of the quantity $\left(\alpha_{1}+\alpha_{2}\right)^{n}$, then

$$
E\left\|\left(\Phi^{n} x\right)_{t}-\left(\Phi^{n} y\right)_{t}\right\|_{C_{r}}^{2} \leq\left(\sum_{j=n}^{2 n} \alpha_{n, j-n+1} \frac{t^{j}}{j !}\right)\|x-y\|_{X_{T, 2}}^{2},
$$

and subsequently, after taking the supremum over $[0, T]$,

$$
\left\|\left(\Phi^{n} x\right)_{t}-\left(\Phi^{n} y\right)_{t}\right\|_{X_{T, 2}}^{2} \leq\left(\sum_{j=n}^{2 n} \alpha_{n, j-n+1} \frac{T^{j}}{j !}\right)\|x-y\|_{X_{T, 2}}^{2}=z_{n}\|x-y\|_{X_{T, 2}}^{2} .
$$

Clearly, $\alpha_{n, j-n+1} \rightarrow 0$ as $n \rightarrow \infty$, for all $j \geq 1$, and $\sum_{j=n}^{2 n} \alpha_{n, j-n+1} \leq 1$, for all $n \geq 1$, since $\alpha_{1}+$ $\alpha_{2}<1$ by assumption. Since $\lim _{j \rightarrow \infty}\left(T^{j} / j !\right)=0$, for all $T$, it follows from [20, Theorem 4 , page 74] that $z_{n} \rightarrow 0$ as $n \rightarrow \infty$. As such, there is an $N$ such that $z_{N}<1$, thereby showing that $\Phi^{N}$ is a strict contraction (cf. (3.15)). Regarding the case when $T>1$, note that $T^{2}>$ $T$, so that continuing inequality (3.12) yields

$$
\begin{aligned}
& E\left\|(\Phi x)_{t}-(\Phi y)_{t}\right\|_{C_{r}}^{2} \\
& \leq T^{2}\left[\alpha_{1} \int_{0}^{t} E\left\|x_{\theta}-y_{\theta}\right\|_{C_{r}}^{2} d \theta+\alpha_{2} \int_{0}^{t} \int_{0}^{\theta} E\left\|x_{\tau}-y_{\tau}\right\|_{C_{r}}^{2} d \tau d \theta\right], \quad 0 \leq t \leq T,
\end{aligned}
$$


so that reasoning as above leads to

$$
\left\|\left(\Phi^{n} x\right)_{t}-\left(\Phi^{n} y\right)_{t}\right\|_{X_{T, 2}}^{2} \leq z_{n} T^{2}\|x-y\|_{X_{T, 2}}^{2}
$$

Thus, we can deduce that there is an $N^{*}$ such that $z_{N^{*}}<1 / T^{2}$, again showing that $\Phi^{N^{*}}$ is a strict contraction. Hence, for a given $\mu \in \mathscr{C}_{\lambda^{2}}$ and $T>0$, $\Phi$ has a unique fixed point $x_{\mu} \in X_{T, 2}$. Since $\left(\Phi x_{\mu}\right)(t)=\phi(t)$, for $-r \leq t \leq 0$ (cf. (3.2)), we conclude that $x_{\mu}$ is a mild solution of (1.1).

To complete the proof, we must show that $\mu$ is, in fact, the probability law of $x_{\mu}$. Toward this end, let $\mathscr{L}\left(x_{\mu}\right)=\left\{\mathscr{L}\left(x_{\mu}(t)\right): t \in[-r, T]\right\}$ represent the probability law of $x_{\mu}$ and define the map $\Psi: \mathscr{C}_{\lambda^{2}} \rightarrow \mathscr{C}_{\lambda^{2}}$ by $\Psi(\mu)=\mathscr{L}\left(x_{\mu}\right)$. It is not difficult to see that $\mathscr{L}\left(x_{\mu}(t)\right) \in \wp_{\lambda^{2}}(H)$, for all $t \in[-r, T]$ since $x_{\mu} \in X_{T, 2}$ and $\phi \in L^{2}\left(\Omega ; C_{r}\right)$. Now, to verify the continuity of the map $t \mapsto \mathscr{L}\left(x_{\mu}(t)\right)$, first let $-r \leq c \leq 0$ and take $|h|>0$ small enough to ensure that $-r \leq c+h \leq 0$. For all such $c$,

$$
E\left\|x_{\mu}(c+h)-x_{\mu}(c)\right\|^{2}=E\|\phi(c+h)-\phi(c)\|^{2} \longrightarrow 0 \quad \text { as } h \longrightarrow 0
$$

by assumption. Next, for $0 \leq c \leq T$, observe that for sufficiently small $|h|>0$,

$$
\begin{aligned}
& E\left\|x_{\mu}(c+h)-x_{\mu}(c)\right\|^{2} \\
& \quad \leq 8\left[E\|(S(c+h)-S(c)) \phi(0)\|^{2}+\sum_{i=1}^{3} E\left\|I_{i}^{x_{\mu}}(c+h)-I_{i}^{x_{\mu}}(c)\right\|^{2}\right] .
\end{aligned}
$$

An argument similar to the one used to verify the continuity of $\Phi$ (cf. (3.7)) can be used to then deduce from (3.19) that

$$
\lim _{h \rightarrow 0} E\left\|x_{\mu}(c+h)-x_{\mu}(c)\right\|^{2}=0 \quad \forall-r \leq c \leq T .
$$

Consequently, since for all $c \in[-r, T]$ and $\varphi \in \mathscr{C}_{\lambda^{2}}$, it is the case that

$$
\begin{aligned}
\mid \int_{H} \varphi & (x)\left(\mathscr{L}\left(x_{\mu}(c+h)\right)-\mathscr{L}\left(x_{\mu}(c)\right)\right)(d x) \mid \\
& =\left|\int_{\Omega}\left[\varphi\left(x_{\mu}(c+h ; \omega)\right)-\varphi\left(x_{\mu}(c ; \omega)\right)\right] d \omega\right| \\
& =\left|E\left[\varphi\left(x_{\mu}(c+h)\right)-\varphi\left(x_{\mu}(c)\right)\right]\right| \\
& \leq\|\varphi\|_{\mathscr{C}} E|| x_{\mu}(c+h)-x_{\mu}(c) \|,
\end{aligned}
$$

and so, we can conclude that

$$
\begin{aligned}
& \rho\left(\mathscr{L}\left(x_{\mu}(c+h)\right), \mathscr{L}\left(x_{\mu}(c)\right)\right) \\
& \quad=\sup _{\|\varphi\|_{\mathscr{G} \leq 1}} \int_{H} \varphi(x)\left(\mathscr{L}\left(x_{\mu}(c+h)\right)-\mathscr{L}\left(x_{\mu}(c)\right)\right)(d x) \longrightarrow 0 \quad \text { as }|h| \longrightarrow 0,
\end{aligned}
$$

for any $c \in[-r, T]$. Hence, $t \mapsto \mathscr{L}\left(x_{\mu}(t)\right)$ is a continuous map, so that $\mathscr{L}\left(x_{\mu}\right) \in \mathscr{C}_{\lambda^{2}}$. This shows the well-definedness of $\Psi$. 
Finally, we show that $\Psi$ has a unique fixed point in $\mathscr{C}_{\lambda^{2}}$. Let $\mu, \nu \in \mathscr{C}_{\lambda^{2}}$ and let $x_{\mu}, x_{\nu}$ be the corresponding mild solutions of (1.1). Standard computations yield

$$
\begin{aligned}
E\left\|\left(x_{\mu}\right)_{t}-\left(x_{\nu}\right)_{t}\right\|_{C_{r}}^{2} \leq & C_{1} \int_{0}^{t} E\left\|x_{\theta}-y_{\theta}\right\|_{C_{r}}^{2} d \theta \\
& +C_{2} \int_{0}^{t} \int_{0}^{\theta} E\left\|x_{\tau}-y_{\tau}\right\|_{C_{r}}^{2} d \tau d \theta+C_{3} D_{T}^{2}(\mu, \nu), \quad 0 \leq t \leq T,
\end{aligned}
$$

where

$$
\begin{aligned}
& C_{1}=16 T M_{S}^{2} M_{f_{1}}^{2}, \\
& C_{2}=16 M_{S}^{2} T\left(M_{f_{2}}^{2} M_{K_{1}}^{2} T+M_{f_{3}}^{2} M_{K_{2}}^{2} L_{f_{3}}^{2}\right), \\
& C_{3}=C_{1} T+C_{2} T^{2} .
\end{aligned}
$$

Hence, applying Lemma 2.3 and then taking the supremum over $[0, T]$ yields

$$
\left\|x_{\mu}-x_{\nu}\right\|_{X_{T, 2}}^{2} \leq \varsigma_{T} D_{T}^{2}(\mu, \nu)
$$

where $\varsigma_{T}=C_{3}\left[1+\left(C_{1}+C_{2}\right) T \exp \left(\left(C_{1}+C_{2}\right) T\right)\right](1+T \exp (T))$. We can choose $T$ small enough so that $\varsigma_{T}<1$; denote such a $T$ by $T_{0}$. Then, since

$$
\rho\left(\mathscr{L}\left(x_{\mu}(t)\right), \mathscr{L}\left(x_{\nu}(t)\right)\right) \leq E\left\|x_{\mu}(t)-x_{\nu}(t)\right\| \quad \forall-r \leq t \leq T,
$$

we have

$$
\begin{aligned}
\|\Psi(\mu)-\Psi(\nu)\|_{\mathscr{C}_{\lambda^{2}}}^{2} & =D_{T_{0}}^{2}(\Psi(\mu), \Psi(\nu)) \leq \sup _{t \in\left[-r, T_{0}\right]} E\left\|x_{\mu}(t)-x_{\nu}(t)\right\|^{2} \\
& =\left\|x_{\mu}-x_{\nu}\right\|_{X_{T, 2}}^{2}<\varsigma_{T_{0}} D_{T_{0}}^{2}(\mu, \nu)
\end{aligned}
$$

by (3.25), so that $\Psi$ is a strict contraction on $\mathscr{C}_{\lambda^{2}}\left(\left[-r, T_{0}\right] ;\left(\wp_{\lambda^{2}}(H), \rho\right)\right)$. Thus, (1.1) has a unique mild solution on $\left[0, T_{0}\right]$ with probability distribution $\mu \in \mathscr{C}_{\lambda^{2}}\left(\left[-r, T_{0}\right]\right.$; $\left.\left(\wp_{\lambda^{2}}(H), \rho\right)\right)$. This process can be repeated in order to extend the solution, by continuity, to the entire interval $[0, T]$ in finitely many steps, thereby completing the proof.

Remark 3.3. If we take $r=0$ and replace $f_{3}:[0, T] \times C_{r} \times \wp_{\lambda^{2}}(H) \rightarrow B L(K, H)$ and $f_{i}$ : $[0, T] \times C_{r} \times \wp_{\lambda^{2}}(H) \rightarrow H(i=1,2)$ by $f_{3}:[0, T] \times H \rightarrow B L(K, H)$ and $f_{i}:[0, T] \times H \rightarrow$ $H(i=1,2)$, respectively, we recover [13, Theorem 2.1] as a corollary of Theorem 3.2. Further, when $K_{1}$ and $K_{2}$ are convolution-type kernels satisfying [18, Assumption 3.2], Theorem 3.2 is a generalization of [18, Theorem 3.3].

Observe that the result obtained in Theorem 3.2 for $0 \leq t \leq T$ with delay from $-r \leq$ $t \leq 0$ can be generalized in the natural way to intervals $s \leq t \leq T$ with $s \geq 0$ and delay from $-r+s \leq t \leq s$, using the $\sigma$-algebra $\mathscr{F}_{s}$ in place of $\mathscr{F}_{0}$ and $\{W(t)-W(s): t \geq s\}$ in the role of the Wiener process. In this translated setting, for any $s-r \leq t \leq T$ and $\phi \in L^{2}\left(\Omega ; C_{r}\right)$ which is $\mathscr{F}_{s}$-measurable independent of $W$ with almost surely continuous paths, there 
exists exactly one mild solution, denoted by $x^{s}(t)$, of

$$
\begin{aligned}
x^{\prime}(t)+A x(t)= & f_{1}\left(t, x_{t}, \mu(t)\right)+\int_{s}^{t} K_{1}(t, \tau) f_{2}\left(\tau, x_{\tau}, \mu(\tau)\right) d \tau \\
& +\int_{s}^{t} K_{2}(t, \tau) f_{3}\left(\tau, x_{\tau}, \mu(\tau)\right) d W(\tau), \quad s \leq t \leq T, \\
x(t)= & \phi(t), \quad s-r \leq t \leq s .
\end{aligned}
$$

Next, we establish the continuous dependence of mild solutions of (1.1) on the initial data and the boundedness of the $p$ th moments. Specifically, we have the following.

Proposition 3.4. Assume (A1)-(A4). Then, for any $\phi, \psi \in L^{2}\left(\Omega ; C_{r}\right)$ which are $\mathscr{F}_{0}$-measurable random variables independent of $W$ with almost surely continuous paths and $0 \leq s \leq$ $s^{\prime} \leq t \leq T$, the following estimates hold.

(i) For each $p \geq 1$, there is a constant $C_{p}>0$ such that

$$
\sup _{t \in[0, T]} E\left\|\left(x_{\phi}\right){ }_{t}\right\|_{C_{r}}^{2 p} \leq C_{p}\left(1+E\|\phi(0)\|_{C_{r}}^{2 p}\right) .
$$

(ii) There exist positive constants $\beta_{1}, \beta_{2}$, and $\beta_{3}$ such that

$$
E\left\|\left(x_{\phi}\right)_{t}-\left(x_{\psi}\right)_{t}\right\|_{C_{r}}^{2} \leq \beta_{1}\left[\|\phi-\psi\|_{L^{2}\left(\Omega ; C_{r}\right)}^{2}+D_{T}^{2}\left(\mu_{x_{\phi}}, \mu_{x_{\psi}}\right)\right] \cdot M^{*}(t),
$$

where $M^{*}(t)=\left[1+\exp (t) \cdot\left(1+\beta_{2} t \exp \left(\left(\beta_{2}+\beta_{3}\right) t\right)\right)\right]$ and $x_{\phi}$ and $x_{\psi}$, respectively, denote the corresponding mild solutions of (1.1). In particular,

$$
E\left\|\left(x_{\phi}\right)_{t}-\left(x_{\psi}\right)_{t}\right\|_{C_{r}}^{2} \leq M^{*}\left(\frac{\beta_{1}}{1-\beta_{1} M^{*}}\right)\|\phi-\psi\|_{L^{2}\left(\Omega ; C_{r}\right)}^{2},
$$

where $M^{*}=\sup _{0 \leq t \leq T} M^{*}(t)$, provided that $1-\beta_{1} M^{*}>0$.

(iii) There is a positive continuous function $\varsigma\left(s, s^{\prime}\right)$ such that $\varsigma\left(s, s^{\prime}\right) \rightarrow 0$ as $\left(s-s^{\prime}\right) \rightarrow 0$ for which

$$
E\left\|\left(x_{\phi}^{s^{\prime}}\right)_{t}-\left(x_{\phi}^{s}\right)_{t}\right\|_{C_{r}}^{2} \leq \varsigma\left(s, s^{\prime}\right) .
$$

Proof. Using the computations leading to (3.23) and then applying Lemma 2.3 yields (i). To see why (ii) holds, let $p \geq 1$ and note that standard computations yield

$$
\begin{aligned}
& E\left\|\left(x_{\phi}\right)_{t}\right\|_{C_{r}}^{2 p} \\
& \leq 8^{p}\left[\sup _{-r \leq \theta \leq 0} E\|S(t+\theta) \phi(0)\|^{2}+\sup _{-r \leq \theta \leq 0} \sum_{i=1}^{3} E\left\|I_{i}^{x}(t+\theta)\right\|^{2}\right]^{p} \\
& \leq 16^{p}\left[M_{S}^{2 p}\left(E\|\phi(0)\|_{C_{r}}^{2}\right)^{p}+C_{1}^{p}\left(\int_{0}^{t}\left[1+E\left\|\left(x_{\phi}\right)_{\theta}\right\|_{C_{r}}^{2}+\|\mu(\theta)\|_{\lambda^{2}}^{2}\right] d \theta\right)^{p}\right. \\
&\left.+C_{2}^{p}\left(\int_{0}^{t} \int_{0}^{\theta}\left[1+E\left\|\left(x_{\phi}\right)_{\tau}\right\|_{C_{r}}^{2}+\|\mu(\tau)\|_{\lambda^{2}}^{2}\right] d \tau d \theta\right)^{p}\right], \quad 0 \leq t \leq T .
\end{aligned}
$$


Applying the Jensen and Hölder inequalities enables us to obtain, for all $0 \leq t \leq T$, that

$$
\begin{aligned}
& \left(E\|\phi(0)\|_{C_{r}}^{2}\right)^{p} \leq E\|\phi(0)\|_{C_{r}}^{2 p}, \\
& \left(\int_{0}^{t}\left[1+E\left\|\left(x_{\phi}\right)_{\theta}\right\|_{C_{r}}^{2}+\|\mu(\theta)\|_{\lambda^{2}}^{2}\right] d \theta\right)^{p} \\
& \quad \leq 3^{p} T^{(p-1) / p} \int_{0}^{t}\left[1+E\left\|\left(x_{\phi}\right)_{\theta}\right\|_{C_{r}}^{2 p}+\|\mu(\theta)\|_{\lambda^{2}}^{2 p}\right] d \theta \\
& \left(\int_{0}^{t} \int_{0}^{\theta}\left[1+E\left\|\left(x_{\phi}\right)_{\tau}\right\|_{C_{r}}^{2}+\|\mu(\tau)\|_{\lambda^{2}}^{2}\right] d \tau d \theta\right)^{p} \\
& \quad \leq 3^{p} T^{(2(p-1)) / p} \int_{0}^{t} \int_{0}^{\theta}\left[1+E\left\|\left(x_{\phi}\right)_{\tau}\right\|_{C_{r}}^{2 p}+\|\mu(\tau)\|_{\lambda^{2}}^{2 p}\right] d \tau d \theta .
\end{aligned}
$$

Using (3.34) in (3.33) and subsequently invoking Lemma 2.3 yields (ii), after a rearrangement of terms. Finally, (iii) easily follows by making use of the (A2)-(A4), together with (3.26). This completes the proof.

We now comment on the Markov and strongly Markov properties of the solutions of (3.28). Precisely, in view of Theorem 3.2 and Proposition 3.4, arguments as in [10, pages 248-255] can be used to show the following.

Proposition 3.5. Under the assumptions of Theorem 3.2 and Proposition 3.4 the mild solutions of (3.28) are Markov and strongly Markov.

\section{Convergence results}

For each $n \geq 1$, consider the Yosida approximation of (1.1) given by

$$
\begin{aligned}
x_{n}^{\prime}(t)+A x_{n}(t)= & n R(n ; A) f_{1}\left(t,\left(x_{n}\right)_{t}, \mu_{n}(t)\right) \\
& +\int_{0}^{t} K_{1}(t, s) n R(n ; A) f_{2}\left(s,\left(x_{n}\right)_{s}, \mu_{n}(s)\right) d s \\
& +\int_{0}^{t} K_{2}(t, s) n R(n ; A) f_{3}\left(s,\left(x_{n}\right)_{s}, \mu_{n}(s)\right) d W(s), \quad 0 \leq t \leq T, \\
x_{n}(t)= & n R(n ; A) \phi(t), \quad-r \leq t \leq 0,
\end{aligned}
$$

where $\mu_{n}(t)$ is the probability law of $x_{n}(t)$ and $R(n ; A)=(I-n A)^{-1}$ is the resolvent operator of $A$. Assuming that (A1)-(A4) hold, one can invoke Theorem 3.2 to deduce that (4.1) has a unique mild solution $x_{n} \in X_{T, 2}$ with probability law $\mu_{n} \in \mathscr{C}_{\lambda^{2}}$. Further, since $x_{n}(t) \in D(A)$, for all $t \in[-r, T]$ (see $[10,26]$ ), it is not difficult to argue that $x_{n}$ is, in fact, a strong solution of (4.1) in the sense of the following definition.

Definition 4.1. A continuous stochastic process $x_{n}:[-r, T] \rightarrow H$ is a strong solution of (4.1) if

(i) $x_{n}(t)$ is $\mathscr{F}_{t}$-adapted, for each $-r \leq t \leq T$,

(ii) $x_{n}(t) \in D(A)$, for almost all $0 \leq t \leq T$, almost surely $[P]$,

(iii) $\int_{0}^{T}\|A x(s)\| d s<\infty$, almost surely $[P]$, 
(iv)

$$
\begin{aligned}
& x_{n}(t)= n R(n ; A) \phi(0) \\
&+\int_{0}^{t} A x_{n}(s) d s+\int_{0}^{t} n R(n ; A) f_{1}\left(s,\left(x_{n}\right)_{s}, \mu_{n}(s)\right) d s \\
&+\int_{0}^{t} n R(n ; A) \int_{0}^{s} K_{1}(s, \tau) f_{2}\left(\tau,\left(x_{n}\right)_{\tau}, \mu_{n}(\tau)\right) d \tau d s \\
&+\int_{0}^{t} n R(n ; A) \int_{0}^{s} K_{2}(s, \tau) f_{3}\left(\tau,\left(x_{n}\right)_{\tau}, \mu_{n}(\tau)\right) d W(\tau) d s \\
& \forall 0 \leq t \leq T, \text { almost surely }[P],
\end{aligned}
$$

(v) $x_{n}(t)=n R(n ; A) \phi(t),-r \leq t \leq 0$, almost surely $[P]$.

Moreover, since a strong solution is also a mild solution, $x_{n}$ can be represented using the variation of parameters formula (cf. Definition 3.1(iii)). The following convergence result holds.

Theorem 4.2. Let $x$ denote the unique mild solution of (1.1) as guaranteed by Theorem 3.2. Then, the sequence of strong solutions of (4.1) converges to $x$ in $X_{T, 2}$ as $n \rightarrow \infty$.

Proof. Observe that

$$
\begin{aligned}
\left\|x_{n}(t+\theta)-x(t+\theta)\right\|^{2} & \\
\leq 8[ & \|(n R(n ; A)-I) S(t+\theta) \phi(0)\|^{2} \\
& +M_{S}^{2} T \int_{0}^{t+\theta}\left\|n R(n ; A) f_{1}\left(s,\left(x_{n}\right)_{s}, \mu_{n}(s)\right)-f_{1}\left(s, x_{s}, \mu(s)\right)\right\|^{2} d s \\
& +M_{S}^{2} M_{K_{1}}^{2} T^{2} \int_{0}^{t+\theta} \int_{0}^{s}\left\|n R(n ; A) f_{2}\left(\tau,\left(x_{n}\right)_{\tau}, \mu_{n}(\tau)\right)-f_{2}\left(\tau, x_{\tau}, \mu(\tau)\right)\right\|^{2} d \tau d s \\
& +M_{K_{2}}^{2} \| \int_{0}^{t+\theta} S(t+\theta-s) \int_{0}^{s}\left[n R(n ; A) f_{3}\left(\tau,\left(x_{n}\right)_{\tau}, \mu_{n}(\tau)\right)\right. \\
& \left.\left.-f_{3}\left(\tau, x_{\tau}, \mu(\tau)\right)\right] d W(\tau) d s \|^{2}\right] \\
=8 & {\left[\|(n R(n ; A)-I) S(t+\theta) \phi(0)\|^{2}+\sum_{i=4}^{6} I_{i}(t+\theta)\right] . }
\end{aligned}
$$

Standard computations lead to

$$
\begin{aligned}
I_{4}(t+\theta) \leq 2 M_{S}^{2} T \int_{0}^{t+\theta}[ & \left\|(n R(n ; A)-I) f_{1}\left(s,\left(x_{n}\right)_{s}, \mu_{n}(s)\right)\right\|^{2} \\
& \left.+2 M_{f_{1}}^{2}\left(\left\|\left(x_{n}\right)_{s}-x_{s}\right\|_{C_{r}}^{2}+\rho^{2}\left(\mu_{n}(s), \mu(s)\right)\right)\right] d s, \quad 0 \leq t \leq T .
\end{aligned}
$$


Further, the triangle inequality and (A2), together, imply

$$
\begin{aligned}
\int_{0}^{t+\theta} & \left\|(n R(n ; A)-I) f_{1}\left(s,\left(x_{n}\right)_{s}, \mu_{n}(s)\right)\right\|^{2} d s \\
\leq 2 \int_{0}^{t+\theta}\|n R(n ; A)-I\|^{2}\left[2 M_{f_{1}}^{2}(\right. & \left\|\left(x_{n}\right)_{s}-x_{s}\right\|_{C_{r}}^{2}+\rho^{2}\left(\mu_{n}(s), \mu(s)\right) \\
& \left.\left.+\left\|f_{1}\left(s, x_{s}, \mu(s)\right)\right\|^{2}\right)\right] d s, \quad 0 \leq t \leq T .
\end{aligned}
$$

The boundedness of $\left\|f_{1}\left(s, x_{s}, \mu(s)\right)\right\|^{2}$ independent of $n$ (which follows from (A2) and Proposition 3.4), together with the strong convergence of $n R(n ; A)-I$ to 0 , enable us to infer that the right-hand side of (4.5) goes to 0 as $n \rightarrow \infty$.

Similar computations lead to

$$
\begin{aligned}
& I_{5}(t+\theta) \leq 2 M_{S}^{2} M_{K_{1}}^{2} T^{2} \int_{0}^{t+\theta} \int_{0}^{s}\left[\left\|(n R(n ; A)-I) f_{2}\left(\tau,\left(x_{n}\right)_{\tau}, \mu_{n}(\tau)\right)\right\|^{2}\right. \\
& \left.+2 M_{f_{2}}^{2}\left(\left\|\left(x_{n}\right)_{\tau}-x_{\tau}\right\|_{C_{r}}^{2}+\rho^{2}\left(\mu_{n}(\tau), \mu(\tau)\right)\right)\right] d \tau d s, \\
& 0 \leq t \leq T \text {, } \\
& I_{6}(t+\theta) \leq 2 M_{S}^{2} M_{K_{2}}^{2} T^{2} \int_{0}^{t+\theta} \int_{0}^{s}\left[\left\|(n R(n ; A)-I) f_{3}\left(\tau,\left(x_{n}\right)_{\tau}, \mu_{n}(\tau)\right)\right\|^{2}\right. \\
& \left.+2 M_{f_{3}}^{2} L_{f_{3}}^{2}\left(\left\|\left(x_{n}\right)_{\tau}-x_{\tau}\right\|_{C_{r}}^{2}+\rho^{2}\left(\mu_{n}(\tau), \mu(\tau)\right)\right)\right] d \tau d s, \\
& 0 \leq t \leq T \text {. }
\end{aligned}
$$

The dominated convergence theorem, together with (A3) and (A4) and the strong convergence of $n R(n ; A)-I$ to 0 , enables us to conclude that the first integrals on the right-hand sides of both (4.6) and (4.7) go to 0 as $n \rightarrow \infty$. Hence, using (4.4), (4.6), and (4.7), together with subsequent estimates in the spirit of (4.5) in (4.3), followed by taking the supremum over $-r \leq \theta \leq 0$ and subsequently taking expectations, gives rise to an inequality of the form

$$
\begin{aligned}
E\left\|\left(x_{n}\right)_{t}-x_{t}\right\|_{C_{r}}^{2} \leq & \bar{\beta}_{1} E\|\phi(0)\|^{2}+\bar{\beta}_{2} \int_{0}^{t} E\left\|\left(x_{n}\right)_{s}-x_{s}\right\|_{C_{r}}^{2} d s \\
& +\bar{\beta}_{3} \int_{0}^{t} \int_{0}^{s} E\left\|\left(x_{n}\right)_{\tau}-x_{\tau}\right\|_{C_{r}}^{2} d \tau d s, \quad 0 \leq t \leq T,
\end{aligned}
$$

where $\bar{\beta}_{i}(i=1,2,3)$ are constant multiples of the quantity $\|n R(n ; A)-I\|^{2}$. Applying Lemma 2.3 and then taking the supremum over $0 \leq t \leq T$ yields

$$
\left\|x_{n}-x\right\|_{X_{T, 2}}^{2} \leq \bar{\beta}_{1}\left[1+T \bar{\beta}_{2} \exp \left(\bar{\beta}_{2} T\right) \cdot\left(1+T \bar{\beta}_{3} \exp \left(\bar{\beta}_{3} T\right)\right)\right] \quad \forall n \geq 1 .
$$

Since the right-hand side of (4.9) goes to 0 as $n \rightarrow \infty$, the conclusion of the theorem now follows.

The following corollary is needed to establish the weak convergence of probability measures (cf. Theorem 4.6). 
Corollary 4.3. The sequence of probability laws $\mu_{n}$ corresponding to the mild solution $x_{n}$ of (4.1) converges in $\mathscr{C}_{\lambda^{2}}$ to the probability law $\mu$ corresponding to the mild solution of (1.1) as $n \rightarrow \infty$.

Proof. This follows from the fact that

$$
\begin{aligned}
D_{T}^{2}\left(\mu_{n}, \mu\right) & =\sup _{[-r, T]} \rho^{2}\left(\mu_{n}(t), \mu(t)\right) \\
& \leq \sup _{[-r, T]} E\left\|\left(x_{n}\right)_{t}-x_{t}\right\|_{C_{r}}^{2} \leq\left\|x_{n}-x\right\|_{X_{T, 2}}^{2} \longrightarrow 0 \quad \text { as } n \longrightarrow \infty
\end{aligned}
$$

for all $0 \leq t \leq T$.

Remark 4.4. We observe for later purposes that Corollary 4.3 implies that

$$
\sup _{n \in \mathbb{N}} \sup _{-r \leq s \leq T}\left\|\mu_{n}(s)\right\|_{\lambda^{2}}^{2}<\infty .
$$

In view of Theorem 4.2 and Corollary 4.3 , the following continuity-type result can be established as in [1]. The details are omitted.

Corollary 4.5. Assume that $E\|\phi(0)\|^{4}<\infty$. Then, for any function $F:[0, T] \times H \rightarrow \mathbb{R}$ satisfying

(i) for each $N \in \mathbb{N}$, there exists a positive continuous function $k_{N}(t)$ such that

$$
|F(t, x)-F(t, y)| \leq k_{N}(t)|x-y| \quad \forall 0 \leq t \leq T,\|x\| \leq N,\|y\| \leq N
$$

(ii) there exists a positive continuous function $l(t)$ such that

$$
|F(t, x)| \leq l(t) \lambda^{2}(x) \quad \forall 0 \leq t \leq T, x \in H,
$$

it holds that

$$
\int_{0}^{T} \int_{H} F(t, x) d\left(\mu_{n}(t)-\mu(t)\right) d t \longrightarrow 0 \text { as } n \longrightarrow \infty .
$$

We now consider the weak convergence of the probability measures induced by the mild solutions of (4.1). Let $P_{x}$ denote the probability measure generated by the mild solution $x$ of (1.1) and $P_{x_{n}}$ the probability measure generated by $x_{n}$ as in (4.1). Also, let $\phi \in L^{4}\left(\Omega, C_{r}\right)$ be an $\mathscr{F}_{0}$-measurable random variable independent of $W$ with almost surely continuous sample paths. Assuming (A1)-(A4), we have the following.

Theorem 4.6.

$$
P_{x_{n}} \stackrel{w}{\longrightarrow} P_{x} \text { as } n \longrightarrow \infty \text {. }
$$

Proof. We will show that $\left\{P_{x_{n}}\right\}_{n=1}^{\infty}$ is relatively compact by using Arzelá-Ascoli. Throughout the proof, $C_{i}$ will denote a suitable positive constant. 
First, we show that $\left\{x_{n}\right\}$ is uniformly bounded in $X_{T, 2}$, that is, $\sup _{n \in \mathbb{N}} \sup _{0 \leq t \leq T}$ $E\left\|\left(x_{n}\right)_{t}\right\|_{C_{r}}^{2}<\infty$. The mild solution $x_{n}$ is given by the variation of parameters formula

$$
\begin{aligned}
x_{n}(t)= & S(t) n R(n ; A) \phi(0)+\int_{0}^{t} S(t-s) n R(n ; A) f_{1}\left(s,\left(x_{n}\right)_{s} \mu_{n}(s)\right) d s \\
& +\int_{0}^{t} \int_{0}^{s} S(s-t) n R(n ; A) f_{2}\left(\tau,\left(x_{n}\right)_{s}, \mu_{n}(s)\right) d \tau d s \\
& +\int_{0}^{t} \int_{0}^{s} S(s-t) n R(n ; A) f_{3}\left(\tau,\left(x_{n}\right)_{s}, \mu_{n}(s)\right) d W(\tau) d s, \quad 0 \leq t \leq T \\
= & S(t) n R(n ; A) \phi(0)+\sum_{i=7}^{9} I_{i}(t), \\
x_{n}(t)= & n R(n ; A) \phi(t), \quad-r \leq t \leq 0 .
\end{aligned}
$$

Let $t \in[0, T]$. For all $-r \leq \theta \leq 0$ for which $t+\theta \leq 0$, we have

$$
\begin{aligned}
\sup _{n \in \mathbb{N}} & \sup _{-r \leq \theta \leq 0}\left\{E\left\|x_{n}(t+\theta)\right\|^{2}:-r \leq t+\theta \leq 0\right\} \\
& =\sup _{n \in \mathbb{N}} \sup _{-r \leq \theta \leq 0}\left\{E\|n R(n ; A) \phi(t+\theta)\|^{2}:-r \leq t+\theta \leq 0\right\}<\infty
\end{aligned}
$$

since $n R(n ; A)$ is contractive for all $n$. Now, let $-r \leq \theta \leq 0$ be such that $0 \leq t+\theta \leq T$. We consider each of the four terms on the right-hand side of (4.16) separately. First

$$
E\|S(t+\theta) n R(n ; A) \phi(0)\|_{C_{r}}^{2} \leq M_{S}^{2} E\|\phi(0)\|_{C_{r}}^{2} .
$$

Using routine arguments, taking into account (A2) and Remark 4.4, leads to

$$
E\left\|I_{7}(t+\theta)\right\|_{C_{r}}^{2} \leq 2 T M_{S}^{2} \bar{M}_{f_{1}}^{2}\left[T C_{1}+\int_{0}^{t+\theta} E\left\|\left(x_{n}\right)_{s}\right\|_{C_{r}}^{2} d s\right] .
$$

In a similar manner, we obtain

$$
E\left\|I_{8}(t+\theta)\right\|_{C_{r}}^{2} \leq 2 M_{S}^{2} T^{2} M_{K_{1}}^{2} \bar{M}_{f_{2}}^{2}\left[T^{2} C_{2}+\int_{0}^{t+\theta} \int_{0}^{s} E\left\|\left(x_{n}\right)_{\tau}\right\|_{C_{r}}^{2} d \tau d s\right],
$$

and using Lemma 2.2,

$$
E\left\|I_{9}(t+\theta)\right\|_{C_{r}}^{2} \leq 2 M_{S}^{2} T^{2} M_{K_{2}}^{2} \bar{M}_{f_{3}}^{2} L_{f_{3}}\left[T^{2} C_{3}+\int_{0}^{t+\theta} \int_{0}^{s} E\left\|\left(x_{n}\right)_{\tau}\right\|_{C_{r}}^{2} d \tau d s\right] .
$$


Combining (4.18), (4.19), (4.20), and (4.21), there exist constants $C_{4}, C_{5}, C_{6}$ independent of $n$ such that

$$
E\left\|\left(x_{n}\right)_{t}\right\|_{C_{r}}^{2} \leq C_{4}+C_{5} \int_{0}^{t} E\left\|\left(x_{n}\right)_{s}\right\|_{C_{r}}^{2} d s+C_{6} \int_{0}^{t} \int_{0}^{s} E\left\|\left(x_{n}\right)\right\|_{C_{r}}^{2} d \tau d s
$$

which, by using Lemma 2.3, shows the uniform boundedness of $\left\{x_{n}\right\}$ in $X_{T, 2}$.

Next, we show equicontinuity. We will show that for every $n \in \mathbb{N}$ and $-r \leq s \leq t \leq T$, as $t-s \rightarrow 0$, we have $E\left\|x_{n}(t)-x_{n}(s)\right\|^{4} \rightarrow 0$ independent of $n$. If $-r \leq s \leq t \leq 0$, note that

$$
E\left\|x_{n}(t)-x_{n}(s)\right\|^{4}=E\|n R(n ; A)[\phi(t)-\phi(s)]\|^{4} \longrightarrow 0
$$

independent of $n$ since $\phi \in L^{4}\left(\Omega, C_{r}\right)$ and $n R(n ; A)$ is contractive, for all $n$. Now consider $0 \leq s \leq t \leq T$. Since $\{S(t)\}$ is a semigroup,

$$
\begin{aligned}
E\|(S(t)-S(s)) n R(n ; A) \phi(0)\|^{4} & \leq E\left(\int_{s}^{t}\|S(\tau) A n R(n ; A) \phi(0)\| d \tau\right)^{4} \\
& \leq M_{S}^{4} M_{A}^{4} E\|\phi(0)\|^{4}(t-s)^{4} .
\end{aligned}
$$

Also,

$$
\begin{gathered}
E\left\|I_{1}(t)-I_{1}(s)\right\|^{4} \\
\leq E\left(\int_{0}^{s}\left\|[S(t-\tau)-S(s-\tau)] n R(n ; A) f_{1}\left(\tau,\left(x_{n}\right)_{t}, \mu_{n}(\tau)\right)\right\| d \tau\right. \\
\left.\quad+\int_{s}^{t}\left\|S(t-\tau) n R(n ; A) f_{1}\left(\tau,\left(x_{n}\right)_{t}, \mu_{n}(\tau)\right)\right\| d \tau\right)^{4} \\
\leq E\left(\int_{0}^{s} \int_{s-\tau}^{t-\tau}\left\|S(u) A n R(n ; A) n R(n ; A) f_{1}\left(\tau,\left(x_{n}\right)_{t}, \mu_{n}(\tau)\right)\right\| d u d \tau\right. \\
\left.\quad+M_{S} \bar{M}_{f_{1}}\left[1+\|x\|_{T, 2}+\sup _{0 \leq t \leq T}\left\|\mu_{n}(t)\right\|_{\lambda^{2}}\right](t-s)\right)^{4} \\
\leq E\left(M_{S} M_{A} T \bar{M}_{f_{1}}\left[1+\|x\|_{T, 2}+\sup _{0 \leq t \leq T}\left\|\mu_{n}(t)\right\|_{\lambda^{2}}\right](t-s)\right. \\
\left.\quad+M_{S} \bar{M}_{f_{1}}\left[1+\|x\|_{T, 2}+\sup _{0 \leq t \leq T}\left\|\mu_{n}(t)\right\|_{\lambda^{2}}\right](t-s)\right)^{4} \\
=C_{7}(t-s)^{4} .
\end{gathered}
$$

Similarly,

$$
E|| I_{8}(t)-\left.I_{8}(s)\right|^{4} \leq C_{8}(t-s)^{4} .
$$


Finally,

$$
\begin{aligned}
& E \| I_{9}(t)- I_{9}(s) \|^{4} \\
&=E \| \int_{0}^{s} \int_{0}^{\sigma}[S(t-s)-S(s-\sigma)] n R(n ; A) K_{2}(\sigma, \tau) f_{3}\left(\tau,\left(x_{n}\right)_{\tau}, \mu_{n}(\tau)\right) d W(\tau) d \sigma \\
& \quad+\int_{s}^{t} \int_{0}^{\sigma} S(t-\sigma) n R(n ; A) K_{2}(\sigma, \tau) f_{3}\left(\tau,\left(x_{n}\right)_{\tau}, \mu_{n}(\tau)\right) d W(\tau) d \sigma \|^{4} \\
&=E \| \int_{0}^{s} \int_{\tau}^{s}[S(t-s)-S(s-\sigma)] n R(n ; A) K_{2}(\sigma, \tau) f_{3}\left(\tau,\left(x_{n}\right)_{\tau} \mu_{n}(\tau)\right) d \sigma d W(\tau) \\
& \quad+\left[\int_{0}^{s} \int_{s}^{t}+\int_{s}^{t} \int_{\tau}^{s}+\int_{s}^{t} \int_{s}^{t}\right] S(t-\sigma) n R(n ; A) K_{2}(\sigma, \tau) \\
& \quad \times f_{3}\left(\tau,\left(x_{n}\right)_{\tau} \mu_{n}(\tau)\right) d \sigma d W(\tau) \|^{4},
\end{aligned}
$$

and hence, as in previous computations, the expression in (4.27) is bounded above by $C_{9}(t-s)^{4}$ independent of $n$. Thus, combining the above estimates coming from (4.24)(4.27), the equicontinuity follows.

Therefore, the family $\left\{P_{x_{n}}\right\}_{n=1}^{\infty}$ is relatively compact by Arzelá-Ascoli, and therefore tight (cf. Section 2). Hence, by Proposition 2.5, the finite-dimensional joint distributions of $P_{x_{n}}$ converge weakly to that of $P_{x}$ and so, by Theorem $2.6, P_{x_{n}} \stackrel{w}{\rightarrow} P_{x}$, as $n \rightarrow \infty$.

\section{Asymptotic stability}

We now consider $(1.1)$ on $[0, \infty)$ rather than on a finite interval, and denote a global mild solution of (1.1) corresponding to $\phi \in L^{p}\left(\Omega ; C_{r}\right)$ by $x(t ; \phi)$, if one should exist. We formulate results regarding the $p$ th-moment exponential stability and almost sure exponential stability of such solutions in the following sense (see $[30,31]$ ).

Definition 5.1. $x(t ; \phi)$ is said to be

(i) pth-moment exponentially stable if there exist positive constants $\lambda$ and $C$ such that for every $\phi \in L^{p}\left(\Omega ; C_{r}\right), E\|x(t ; \phi)\|_{C_{r}}^{p} \leq C E\|\phi\|_{C_{r}}^{p} \exp (-\lambda t)$, for all $t \geq 0$, almost surely $[P]$,

(ii) almost surely exponentially stable if there exists a positive constant $\lambda$ such that for every $\phi \in L^{p}\left(\Omega ; C_{r}\right)$, there is a finite random variable $\beta$ such that $\|x(t ; \phi)\| \leq$ $\beta \exp (-\lambda t)$, for all $t \geq 0$, almost surely $[P]$, (or equivalently, $\overline{\lim }_{t \rightarrow \infty}(1 / t) \log \| x(t$; $\phi) \| \leq-\lambda$, almost surely $[P])$.

Throughout this section, we will assume that $f_{i}(i=1,2,3)$, now defined on $[0, \infty) \times$ $C_{r} \times \wp_{\lambda^{2}}(H)$, satisfy the growth conditions in (A2)-(A4) globally on their respective domains, and that the kernels $K_{1}(t, s)$ and $K_{2}(t, s)$ are defined for $0 \leq s \leq t<\infty$ and are globally bounded. In addition, assume that

(A5) $\|S(t)\| \leq \bar{M} \exp (-\alpha t)$, for all $t \geq 0$, for some constants $\bar{M} \geq 1$ and $\alpha>0$. 
Also, replace $\mathscr{C}_{\lambda^{2}}$ by $\mathscr{C}_{\lambda^{2}, \infty}$, the space of all bounded continuous $\wp_{\lambda^{2}}(H)$-valued functions defined on $[0, \infty)$, equipped with the metric

$$
D_{\infty}\left(\nu_{1}, v_{2}\right)=\sup _{t \in[0, \infty)} \rho\left(\nu_{1}(t), \nu_{2}(t)\right), \quad v_{1}, v_{2} \in \mathscr{C}_{\lambda^{2}, \infty}
$$

A mild solution of $(1.1)$ on $[0, \infty)$ is a continuous process $x$ that satisfies Definition 3.1, for each $T>0$. One can use the same approach used in the proof of Theorem 3.2 to verify that under the above modified hypotheses, the mild solution of (1.1) can be extended to $[0, \infty)$ with probability distribution $\mu \in \mathscr{C}_{\lambda^{2}, \infty}$. In order to establish stability results in the spirit of Definition 5.1 of such a mild solution, however, the growth conditions imposed in (A2)-(A4) must be strengthened. Precisely, we assume instead that

(A6) $f_{i}:[0, \infty) \times C_{r} \times \wp_{\lambda^{2}}(H) \rightarrow H(i=1,2)$ is a continuous map satisfying

$$
\left\|f_{i}(t, \varphi, \mu(t))\right\| \leq \bar{N}_{f_{i}} \exp \left(-\beta_{f_{i}} t\right)\left[1+\|\varphi\|_{C_{r}}+\|\mu(t)\|_{\lambda^{2}}\right]
$$

globally on $[0, \infty) \times C_{r} \times \wp_{\lambda^{2}}(H)$, for some positive constants $\bar{N}_{f_{i}}$ and $\beta_{f_{i}}$, (A7) $f_{3}:[0, \infty) \times C_{r} \times \wp_{\lambda^{2}}(H) \rightarrow B L(K, H)$ is a continuous map satisfying

$$
\left\|f_{3}(t, \varphi, \mu(t))\right\|_{B L(K, H)} \leq \bar{N}_{f_{3}} \exp \left(-\beta_{f_{3}} t\right)\left[1+\|\varphi\|_{C_{r}}+\|\mu(t)\|_{\lambda^{2}}\right]
$$

globally on $[0, \infty) \times C_{r} \times \wp_{\lambda^{2}}(H)$, for some positive constants $\bar{N}_{f_{3}}$ and $\beta_{f_{3}}$,

(A8) $\left\{K_{1}(t, s): 0 \leq s \leq t<\infty\right\} \cup\left\{K_{2}(t, s): 0 \leq s \leq t<\infty\right\} \subset B L(H, H)$ such that $\left\|K_{1}(t, s)\right\|_{B L(H, H)} \leq \bar{M}_{K_{1}}$ and $\left\|K_{2}(t, s)\right\|_{B L(H, H)} \leq \bar{M}_{K_{2}}$, for all $0 \leq s \leq t<\infty$, for some positive constants $\bar{M}_{K_{1}}$ and $\bar{M}_{K_{2}}$.

Conditions of this type have been imposed by others in analogous situations (e.g., see, $[30,31])$. Throughout the proofs of the results in this section (which utilize the strategy employed in $[30,31]), \varsigma_{i}$ will denote a positive constant depending only on $\alpha, r, \sup _{0 \leq \theta<\infty}$ $\|\mu(\theta)\|_{\lambda}^{p}$, and the growth constants. The first result is as follows.

Theorem 5.2. Assume that (A5)-(A8) hold. Then, the corresponding mild solution of (1.1) on $[0, \infty)$ is pth-moment exponentially stable, provided that $\alpha>2^{1 / p} 3 M_{S} \bar{N}_{f_{1}}$ and $\beta_{f_{i}}>\varepsilon_{0}$ $(i=1,2,3)$ for a positive constant $\varepsilon_{0}$ prescribed later.

Proof. Let $p \geq 1$ and $\phi \in L^{p}\left(\Omega ; C_{r}\right)$ be an $\mathscr{F}_{0}$-measurable random variable independent of $W$ with almost surely continuous paths. For a fixed $t \geq 0$, we have

$$
E\left\|x_{t}\right\|_{C_{r}}^{p} \leq 4^{p} \sup _{-r \leq \theta \leq 0}\left[E\|S(t+\theta) \phi(0)\|^{p}+\sum_{i=1}^{3} E\left\|I_{i}^{x}(t+\theta)\right\|^{p}\right] .
$$

Using (A5) yields

$$
E\|S(t+\theta) \phi(0)\|^{p} \leq \bar{M}^{p} \exp (p \alpha r) \exp (-p \alpha t) E\|\phi(0)\|_{C_{r}}^{p}=\varsigma_{1} \exp (-p \alpha t) .
$$


Next, an application of the Hölder inequality, followed by (A6), gives us

$$
\begin{aligned}
& E\left\|I_{1}^{x}(t+\theta)\right\|^{p} \\
& \leq \bar{M}^{p} E\left[\left(\int_{0}^{t+\theta} \exp (-\alpha(t+\theta-s)) d s\right)^{(p-1)}\right. \\
& \left.\cdot \int_{0}^{t+\theta} \exp (-\alpha(t+\theta-s))\left\|f_{1}\left(s, x_{s}, \mu(s)\right)\right\|^{p} d s\right] \\
& \leq \varsigma_{2} \exp (-\alpha(t+\theta)) \int_{0}^{t+\theta} \exp (\alpha s) E\left\|x_{s}\right\|_{C_{r}}^{p} d s \\
& +\varsigma_{3} \int_{0}^{t+\theta} \exp (-\alpha(t+\theta-s)) \exp \left(-\beta_{f_{1}} s\right) d s .
\end{aligned}
$$

Similarly, we obtain

$$
E\left\|I_{2}^{x}(t+\theta)\right\|^{p} \leq \frac{\left(\bar{M} M_{K_{1}}\right)^{p}}{\alpha^{p-1}} E\left[\int_{0}^{t+\theta} \exp (-\alpha(t+\theta-s))\left(\int_{0}^{s}\left\|f_{2}\left(\tau, x_{\tau}, \mu(\tau)\right)\right\| d \tau\right)^{p} d s\right],
$$

and using (A6), together with the Young and Hölder inequalities, further yields

$$
\begin{aligned}
& \left(\int_{0}^{s}\left\|f_{2}\left(\tau, x_{\tau}, \mu(\tau)\right)\right\| d \tau\right)^{p} \\
& \leq\left(2 \bar{N}_{f_{2}}\right)^{p}[ \\
& {\left[\frac{p-1}{p} \beta_{f_{2}}\right)^{p-1} \int_{0}^{s}\left\|x_{\tau}\right\|_{C_{r}}^{p} d \tau+\frac{2^{p}}{\left(\beta_{f_{2}}\right)^{p-1}}\left(1+\sup _{s \geq 0}\|\mu(s)\|_{\lambda^{2}}^{p}\right)} \\
& \left.\quad \times \int_{0}^{s} \exp \left(-\beta_{f_{2}} \tau\right) d \tau\right] .
\end{aligned}
$$

Then, using (5.8) in (5.7) leads to the subsequent estimate

$$
\begin{aligned}
E\left\|I_{2}^{x}(t+\theta)\right\|^{p} \leq & \varsigma_{4} \exp (-\alpha(t+\theta)) \int_{0}^{t+\theta} \int_{0}^{s} \exp (\alpha s) E\left\|x_{\tau}\right\|_{C_{r}}^{p} d \tau d s \\
& +\varsigma_{5} \int_{0}^{t+\theta} \exp (-\alpha(t+\theta-s)) \int_{0}^{s} \exp \left(-\beta_{f_{2}} \tau\right) d \tau d s .
\end{aligned}
$$

In a completely analogous manner (with the additional help of Itó's formula), we arrive at

$$
\begin{aligned}
E\left\|I_{3}^{x}(t+\theta)\right\|^{p} \leq & \varsigma_{6} \exp (-\alpha(t+\theta)) \int_{0}^{t+\theta} \int_{0}^{s} \exp (\alpha s) E\left\|x_{\tau}\right\|_{C_{r}}^{p} d \tau d s \\
& +\varsigma_{7} \int_{0}^{t+\theta} \exp (-\alpha(t+\theta-s)) \int_{0}^{s} \exp \left(-\beta_{f_{3}} \tau\right) d \tau d s .
\end{aligned}
$$


Combining (5.5), (5.6), (5.9), and (5.10), we conclude that

$$
\begin{aligned}
E\|x(t+\theta)\|^{p} \leq & \varsigma_{1} \exp (-p \alpha t)+\varsigma_{2} \exp (-\alpha(t+\theta)) \int_{0}^{t+\theta} \exp (\alpha s) E\left\|x_{s}\right\|_{C_{r}}^{p} d s \\
& +\left(\varsigma_{4}+\varsigma_{6}\right) \exp (-\alpha(t+\theta)) \int_{0}^{t+\theta} \int_{0}^{s} \exp (\alpha s) E\left\|x_{\tau}\right\|_{C_{r}}^{p} d \tau d s \\
& +\varsigma_{3} \int_{0}^{t+\theta} \exp (-\alpha(t+\theta-s)) \exp \left(-\beta_{f_{1}} s\right) d s \\
& +\int_{0}^{t+\theta} \exp (-\alpha(t+\theta-s)) \int_{0}^{s}\left[\varsigma_{5} \exp \left(-\beta_{f_{2}} \tau\right)+\varsigma_{7} \exp \left(-\beta_{f_{3}} \tau\right)\right] d \tau d s .
\end{aligned}
$$

Choose $0<\alpha / 2<\varepsilon_{0}<\alpha$ such that

$$
2\left(2 M_{S}\right)^{p}\left(\frac{p-1}{\alpha p}\right)^{p-1} \exp \left(\varepsilon_{0} r\right)\left[\frac{\beta_{f_{3}}\left(\bar{N}_{f_{2}} \beta_{f_{2}}\right)^{p}+\beta_{f_{2}}\left(\bar{N}_{f_{3}} \beta_{f_{3}} L_{f_{3}}\right)^{p}}{\beta_{f_{2}} \beta_{f_{3}}}\right]<\varepsilon_{0}\left(\alpha-\varepsilon_{0}\right) .
$$

For $\varepsilon_{0}$ as above and $T$ large enough, multiplying (5.11) by $\exp \left(\varepsilon_{0} t\right)$ and integrating over $(0, T)$ yields

$$
\int_{0}^{T} \exp \left(\varepsilon_{0} t\right) E\|x(t+\theta)\|^{p} d t \leq \varsigma_{1} \int_{0}^{T} \exp \left(\varepsilon_{0} t-p \alpha t\right) d t+\sum_{i=1}^{4} J_{i}(T) .
$$

Applying Fubini's theorem yields

$$
J_{1}(T)=\varsigma_{2}\left[\int_{0}^{T+\theta} \int_{s-\theta}^{T} h_{1}(t, s) d t d s+\int_{\theta}^{0} \int_{0}^{s-\theta} h_{1}(t, s) d t d s\right]=\varsigma_{2}\left[J_{1}^{*}(T)+J_{2}^{*}(T)\right],
$$

where

$$
h_{1}(t, s)=\exp \left(\varepsilon_{0} t-\alpha(t+\theta)\right) \exp (\alpha s) E\left\|x_{s}\right\|_{C_{r}}^{p} .
$$

Elementary computations give rise to

$$
\begin{aligned}
J_{1}^{*}(T) \leq & \frac{\exp (-\alpha \theta)}{\alpha-\varepsilon_{0}} \\
& \times \int_{0}^{T}\left[\exp \left(\left(\varepsilon_{0}-\alpha\right)(s-\theta)\right)-\exp \left(\left(\varepsilon_{0}-\alpha\right) T\right)\right] \exp (\alpha s) E\left\|x_{s}\right\|_{C_{r}}^{p} d s \\
\leq & \frac{\exp \left(\varepsilon_{0} r\right)}{\alpha-\varepsilon_{0}} \int_{0}^{T} \exp \left(\varepsilon_{0} s\right) E\left\|x_{s}\right\|_{C_{r}}^{p} d s, \\
J_{2}^{*}(T) \leq & \frac{\exp (-\alpha \theta)}{\alpha-\varepsilon_{0}} \int_{s}^{0}\left[1-\exp \left(\left(\varepsilon_{0}-\alpha\right)(s-\theta)\right)\right] \exp (\alpha s) E\left\|x_{s}\right\|_{C_{r}}^{p} d s \\
& \leq \frac{\exp (\alpha r)}{\alpha-\varepsilon_{0}} \int_{s}^{0} \exp (\alpha s) E\left\|x_{s}\right\|_{C_{r}}^{p} d s,
\end{aligned}
$$


so that using (5.16) and (5.17) in (5.14) yields

$$
J_{1}(T) \leq \varsigma_{2}\left[\frac{\exp \left(\varepsilon_{0} r\right)}{\alpha-\varepsilon_{0}} \int_{0}^{T} \exp \left(\varepsilon_{0} s\right) E\left\|x_{s}\right\|_{C_{r}}^{p} d s+\frac{\exp (\alpha r)}{\alpha-\varepsilon_{0}} \int_{s}^{0} \exp (\alpha s) E\left\|x_{s}\right\|_{C_{r}}^{p} d s\right] .
$$

Similarly,

$$
\begin{aligned}
J_{2}(T) & =\left(\varsigma_{4}+\varsigma_{6}\right)\left[\int_{s}^{0} \int_{s}^{\tau} \int_{0}^{s-\theta} h_{2}(t, s, \tau) d t d s d \tau+\int_{0}^{T+\theta} \int_{\tau}^{T+\theta} \int_{0}^{s-\theta} h_{2}(t, s, \tau) d t d s d \tau\right] \\
& =\left(\varsigma_{4}+\varsigma_{6}\right)\left[J_{3}^{*}(T)+J_{4}^{*}(T)\right],
\end{aligned}
$$

where

$$
h_{2}(t, s, \tau)=\exp \left(\varepsilon_{0} t-\alpha(t+\theta)\right) \exp (\alpha s) E\left\|x_{\tau}\right\|_{C_{r}}^{p} .
$$

One can easily verify that

$$
\begin{aligned}
& J_{3}^{*}(T) \leq \frac{\exp (\alpha r)}{\alpha\left(\alpha-\varepsilon_{0}\right)} \int_{s}^{0} \exp (\alpha s) E\left\|x_{s}\right\|_{C_{r}}^{p} d s, \\
& J_{4}^{*}(T) \leq \frac{\exp \left(\varepsilon_{0} r\right)}{\varepsilon_{0}\left(\alpha-\varepsilon_{0}\right)} \int_{0}^{T} \exp \left(\varepsilon_{0} s\right) E\left\|x_{s}\right\|_{C_{r}}^{p} d s .
\end{aligned}
$$

Hence, using (5.21) and (5.22) in (5.19), we obtain

$$
\begin{aligned}
J_{2}(T) \leq\left(\varsigma_{4}+\varsigma_{6}\right)\left[\frac{\exp (\alpha r)}{\alpha\left(\alpha-\varepsilon_{0}\right)} \int_{s}^{0} \exp (\alpha s) E\left\|x_{s}\right\|_{C_{r}}^{p} d s\right. \\
\left.+\frac{\exp \left(\varepsilon_{0} r\right)}{\varepsilon_{0}\left(\alpha-\varepsilon_{0}\right)} \int_{0}^{T} \exp \left(\varepsilon_{0} s\right) E\left\|x_{s}\right\|_{C_{r}}^{p} d s\right] .
\end{aligned}
$$

Taking the supremum over $-r \leq \theta \leq 0$ in (5.13) (taking into account the continuity) and then substituting (5.18) and (5.23) gives us

$$
\begin{aligned}
\int_{0}^{T} \exp \left(\varepsilon_{0} t\right) E\left\|x_{t}\right\|_{C_{r}}^{p} d t \leq & \varsigma_{1} \int_{0}^{T} \exp \left(\varepsilon_{0} t-p \alpha t\right) d t+\left[\varsigma_{2}+\frac{1}{\varepsilon_{0}\left(\varsigma_{4}+\varsigma_{6}\right)}\right] \\
& \cdot \frac{\exp \left(\varepsilon_{0} r\right)}{\varepsilon_{0}\left(\alpha-\varepsilon_{0}\right)} \cdot \int_{0}^{T} \exp \left(\varepsilon_{0} s\right) E\left\|x_{s}\right\|_{C_{r}}^{p} d s \\
& +\left[\varsigma_{2}+\frac{1}{\alpha\left(\varsigma_{4}+\varsigma_{6}\right)}\right] \cdot \frac{\exp (\alpha r)}{\alpha\left(\alpha-\varepsilon_{0}\right)} \\
& \times \int_{s}^{0} \exp (\alpha s) E\left\|x_{s}\right\|_{C_{r}}^{p} d s+J_{3}(T)+J_{4}(T) .
\end{aligned}
$$


Now, let $T \rightarrow \infty$ in (5.24). The choice of $\varepsilon_{0}$ and $\alpha$ now enables us to conclude that

$$
\begin{aligned}
\int_{0}^{\infty} \exp \left(\varepsilon_{0} t\right) E\left\|x_{t}\right\|_{C_{r}}^{p} d t \\
\leq \frac{1}{1-\left(\exp \left(\varepsilon_{0} r\right) /\left(\alpha-\varepsilon_{0}\right)\right)\left[\varsigma_{2}+\left(1 /\left(\varepsilon_{0}\left(\varsigma_{4}+\varsigma_{6}\right)\right)\right)\right]} \\
\cdot\left[\varsigma_{1} \int_{0}^{\infty} \exp \left(\varepsilon_{0} t-p \alpha t\right) d t+\left[\varsigma_{2}+\frac{1}{\alpha\left(\varsigma_{4}+\varsigma_{6}\right)}\right]\right. \\
\left.\quad \cdot \frac{\exp (\alpha r)}{\alpha-\varepsilon_{0}} \int_{s}^{0} \exp (\alpha s) E\left\|x_{s}\right\|_{C_{r}}^{p} d s+J_{3}(\infty)+J_{4}(\infty)\right] \\
=C\left(p, \varepsilon_{0}, \phi\right)<\infty,
\end{aligned}
$$

where $J_{i}(\infty)=\lim _{T \rightarrow \infty} J_{i}(T)(i=3,4)$. Hence, we can deduce from $(5.11)$ that

$$
\begin{aligned}
E\left\|x_{t}\right\|_{C_{r}}^{p} \leq \exp \left(-\varepsilon_{0} t\right)[ & \varsigma_{1}+\varsigma_{2} \exp \left(\varepsilon_{0} r\right) C\left(p, \varepsilon_{0}, \phi\right) \\
& +\left(\varsigma_{4}+\varsigma_{6}\right) \exp \left(\varepsilon_{0} r\right) \int_{0}^{t+\theta} \int_{0}^{s} \exp (\alpha s) E\left\|x_{\tau}\right\|_{C_{r}}^{p} d \tau d s \\
& \left.+I_{6}(\infty)+I_{7}(\infty)\right],
\end{aligned}
$$

where $I_{i}(\infty)=\lim _{t \rightarrow \infty} I_{i}(t)(i=6,7)$. (Note that $I_{6}(\infty)+I_{7}(\infty)<\infty$ by choice of $\beta_{f_{i}}$.) Using a similar Fubini-type argument and noting the choice of $\varepsilon_{0}$ enables us to obtain

$$
\int_{0}^{t+\theta} \int_{0}^{s} \exp (\alpha s) E\left\|x_{\tau}\right\|_{C_{r}}^{p} d \tau d s \leq \frac{1}{\alpha} \int_{0}^{\infty} \exp \left(2 \varepsilon_{0}\right) E\left\|x_{s}\right\|_{C_{r}}^{p} d s .
$$

An argument similar to the one leading to (5.25) can then be used to finally conclude from (5.26) and (5.27) that, in fact,

$$
E\left\|x_{t}\right\|_{C_{r}}^{p} \leq C^{*} \exp \left(-\varepsilon_{0} t\right) \quad \forall t \geq 0
$$

as desired.

The second stability result is as follows.

Theorem 5.3. If the mild solution of (1.1) on $[0, \infty)$ is pth-moment exponentially stable, then it is almost surely exponentially stable, provided that the assumptions of Theorem 5.2 are satisfied.

Proof. Let $p \geq 1$ and $k \in \mathbb{N}$ be fixed. First, we claim that

$$
E\left(\sup _{k-1 \leq t \leq k}\|x(t)\|^{p}\right) \leq C \exp \left(-\varepsilon_{0}(k-1)\right)
$$




\section{Stochastic delay equations}

for some positive constant $C$. To see this, note that the semigroup property enables us to write

$$
\begin{aligned}
x(t)= & S(t-(k-1)) x(k-1)+\int_{k-1}^{t} S(t-s) f_{1}\left(s, x_{s}, \mu(s)\right) d s \\
& +\int_{k-1}^{t} S(t-s) \int_{0}^{s} K_{1}(s, \tau) f_{2}\left(\tau, x_{\tau}, \mu(\tau)\right) d \tau d s \\
& +\int_{k-1}^{t} S(t-s) \int_{0}^{s} K_{2}(s, \tau) f_{3}\left(\tau, x_{\tau}, \mu(\tau)\right) d W(\tau) d s, \quad t \geq 0, \\
= & \sum_{i=10}^{13} I_{i}(t), \quad t \geq 0,
\end{aligned}
$$

and subsequently,

$$
E\left(\sup _{k-1 \leq t \leq k}\|x(t)\|^{p}\right) \leq 8^{p} \sum_{i=10}^{13} E\left(\sup _{k-1 \leq t \leq k}\left\|I_{i}(t)\right\|^{p}\right) .
$$

Observe that (A5), together with Theorem 5.2, yields

$$
E\left(\sup _{k-1 \leq t \leq k}\left\|I_{10}(t)\right\|^{p}\right) \leq \bar{M}^{p} E\left\|x_{k-1}\right\|_{C_{r}}^{p} \leq C_{1} \exp \left(-\varepsilon_{0}(k-1)\right)
$$

for some positive constant $C_{1}$. Next, using (A6) and the Hölder inequality, and arguing as in (5.7) and (5.8), we arrive at (by choice of $\beta_{f_{1}}$ )

$$
\begin{aligned}
E\left(\sup _{k-1 \leq t \leq k}\left\|I_{11}(t)\right\|^{p}\right) \\
\leq \bar{M}^{p} E\left(\sup _{k-1 \leq t \leq k} \int_{k-1}^{t}\left\|f_{1}\left(s, x_{s}, \mu(s)\right)\right\|^{p} d s\right) \\
\leq\left(3 \bar{M} \bar{N}_{f_{1}}\right)^{p}\left(\frac{p-1}{\alpha p}\right)^{p-1} \\
\quad \times\left[\int_{k-1}^{k} \exp \left(-\beta_{f_{1}} s\right)\left[1+\sup _{s \geq 0}\|\mu(s)\|_{\lambda^{2}}^{p}\right] d s+\int_{k-1}^{k} \exp \left(-\beta_{f_{1}} s\right) E\left\|x_{s}\right\|_{C_{r}}^{p} d s\right] \\
\leq C_{2} \exp \left(-\varepsilon_{0}(k-1)\right) .
\end{aligned}
$$


A similar computation (using (A7) and the choice of $\beta_{f_{2}}$ ) gives us

$$
\begin{aligned}
E\left(\sup _{k-1 \leq t \leq k}\left\|I_{12}(t)\right\|^{p}\right) \\
\leq\left(2 \bar{M} \bar{M}_{K_{1}} \bar{N}_{f_{2}}\right)^{p}\left(\frac{p-1}{\alpha p}\right)^{p-1} \\
\times E\left(\sup _{k-1 \leq t \leq k} \int_{k-1}^{t} \exp (-\alpha(t-s))\right. \\
\left.\quad \cdot\left[\left(\frac{1+\sup _{s \geq 0}\|\mu(s)\|_{\lambda^{2}}}{\beta_{f_{2}}}\right)^{p}+\left(\frac{p-1}{p \beta_{f_{2}}}\right)^{p-1} \int_{0}^{s}\left\|x_{\tau}\right\|_{C_{r}}^{p} d \tau\right] d s\right) \\
\leq C_{3} \cdot \frac{1}{\alpha} \sup _{k-1 \leq t \leq k}(\exp (-\alpha(t-k+1))-1) \\
+C_{4} C^{*} \cdot \frac{1}{\varepsilon_{0}} \sup _{k-1 \leq t \leq k} \int_{k-1}^{t}\left(1-\exp \left(-\varepsilon_{0} s\right)\right) \exp (-\alpha(t-s)) d s .
\end{aligned}
$$

Since $\varepsilon_{0}<\alpha$, the following hold:

$$
\begin{gathered}
\sup _{k-1 \leq t \leq k}(\exp (-\alpha(t-k+1))-1) \leq \exp \left(-\varepsilon_{0}(k-1)\right), \\
\int_{k-1}^{t}\left(1-\exp \left(-\varepsilon_{0} s\right)\right) \exp (-\alpha(t-s)) d s \leq \exp \left(-\varepsilon_{0}(k-1)\right) .
\end{gathered}
$$

Using (5.35) and (5.36) in (5.34) then yields

$$
E\left(\sup _{k-1 \leq t \leq k}\left\|I_{12}(t)\right\|^{p}\right) \leq C_{5} \exp \left(-\varepsilon_{0}(k-1)\right) .
$$

Similarly, one can argue that

$$
E\left(\sup _{k-1 \leq t \leq k}\left\|I_{13}(t)\right\|^{p}\right) \leq C_{6} \exp \left(-\varepsilon_{0}(k-1)\right),
$$

and hence, using (5.32), (5.33), (5.37), and (5.38) in (5.31) enables us to infer that for each $k \geq 1,(5.29)$ holds, for some positive constant $C$ independent of $k$.

Now, let $0<\lambda<\varepsilon_{0}$ be arbitrary and let $E_{k}$ denote the event

$$
E_{k} \equiv\left\{\omega: \sup _{k-1 \leq t \leq k}\|x(t ; \omega)\|^{p}>\exp \left(-\left(\varepsilon_{0}-\lambda\right)(k-1)\right)\right\} .
$$

Observe that for each $k \geq 1$,

$$
\begin{aligned}
P\left(E_{k}\right) & \leq \exp \left(\left(\varepsilon_{0}-\lambda\right)(k-1)\right) E\left(\sup _{k-1 \leq t \leq k}\|x(t ; \omega)\|^{p}\right) \\
& \leq C \exp (-\lambda(k-1)) \quad(\text { by }(5.29)),
\end{aligned}
$$


so that $\sum_{k=1}^{\infty} P\left(E_{k}\right)<\infty$. Thus, Borel-Cantelli [10] implies that for almost all $\omega \in \Omega$,

$$
\sup _{k-1 \leq t \leq k}\|x(t ; \omega)\|^{p} \leq \exp \left(-\left(\varepsilon_{0}-\lambda\right)(k-1)\right)
$$

holds for all but possibly finitely many $k$. As such, there is a $k_{0} \geq 1$ for which (5.41) holds almost surely $[P]$, for all $k \geq k_{0}$. It then immediately follows that

$$
\frac{1}{t} \log \|x(t)\|^{p} \leq-\frac{\varepsilon_{0}-\lambda}{p} \quad \text { a.s. }[P], k \geq k_{0},
$$

and subsequently,

$$
\varlimsup_{t \rightarrow \infty} \frac{1}{t} \log \|x(t)\|^{p} \leq-\frac{\varepsilon_{0}-\lambda}{p} \quad \text { a.s. }[P] .
$$

Since $\lambda>0$ was arbitrary, the desired conclusion follows from (5.43). (Note that $-\varepsilon_{0} / p$ is an upper bound for the almost sure Lyapunov exponent of the mild solution.)

\section{Examples}

Example 6.1. Let $\mathscr{D}$ be a bounded domain in $\mathbb{R}^{N}$ with smooth boundary $\partial \mathscr{D}$. Consider the following initial-boundary value problem:

$$
\begin{aligned}
\frac{\partial x}{\partial t}(t, z)= & \Delta_{z} x(t, z)+F_{1}(t, z, x(t-r, z))+\int_{L^{2}(\mathscr{D})} F_{2}(t, z, y) \mu(t, z)(d y) \\
& +\int_{0}^{t} a(t, s) g(s, z, x(s-r, z)) d \beta(s) \quad \text { a.e. on }(0, T) \times \mathscr{D}, \\
x(t, z)= & 0 \quad \text { a.e. on }(0, T) \times \partial \mathscr{D}, \\
x(t, z)= & \xi(t, z), \quad-r \leq t \leq 0, \text { a.e. on } \mathscr{D},
\end{aligned}
$$

where $x:[0, T] \times \mathscr{D} \rightarrow \mathbb{R}, F_{1}:[0, T] \times \mathscr{D} \times \mathbb{R} \rightarrow \mathbb{R}, F_{2}:[0, T] \times \mathscr{D} \times L^{2}(\mathscr{D}) \rightarrow L^{2}(\mathscr{D}), \mu(t, \cdot)$ $\in \wp_{\lambda^{2}}\left(L^{2}(\mathscr{D})\right)$ is the probability law of $x(t, \cdot), a: \Delta \rightarrow \mathbb{R}, g:[0, T] \times \mathscr{D} \times \mathbb{R} \rightarrow B L\left(\mathbb{R}^{N}\right.$, $\left.L^{2}(\mathscr{D})\right), \beta$ is a standard $N$-dimensional Brownian motion, and $\xi:[0, T] \times \mathscr{D} \rightarrow \mathbb{R}$. We impose the following conditions.

(A9) $F_{1}$ satisfies the Caratheodory conditions (i.e., measurable in $(t, z)$ and continuous in the third variable) such that

(i) $\left|F_{1}(t, y, z)\right| \leq \bar{M}_{F_{1}}[1+|z|]$, for all $0 \leq t \leq T, y \in \mathscr{D}, z \in \mathbb{R}$, and some $\bar{M}_{F_{1}}>0$,

(ii) $\left|F_{1}\left(t, y, z_{1}\right)-F_{1}\left(t, y, z_{2}\right)\right| \leq M_{F_{1}}\left|z_{1}-z_{2}\right|$, for all $0 \leq t \leq T, y \in \mathscr{D}, z_{1}, z_{2} \in \mathbb{R}$, and some $M_{F_{1}}>0$.

(A10) $F_{2}$ satisfies the Caratheodory conditions and

(i) $\left\|F_{2}(t, y, z)\right\|_{L^{2}(\mathscr{D})} \leq \bar{M}_{F_{2}}\left[1+\|z\|_{L^{2}(\mathscr{D})}\right]$, for all $0 \leq t \leq T, y \in \mathscr{D}, z \in L^{2}(\mathscr{D})$, and some $\bar{M}_{F_{2}}>0$,

(ii) $F_{2}(t, y, \cdot): L^{2}(\mathscr{D}) \rightarrow L^{2}(\mathscr{D})$ is in $\mathscr{C}$, for each $0 \leq t \leq T, y \in \mathscr{D}$.

(A11) $a \in L^{\infty}(0, T)^{2}$. 
(A12) $g$ satisfies the Caratheodory conditions and

(i) $\|g(t, y, z)\|_{B L\left(\mathbb{R}^{N}, L^{2}(\mathscr{D})\right)} \leq \bar{M}_{g}[1+|z|]$, for all $0 \leq t \leq T, y \in \mathscr{D}, z \in \mathbb{R}$, and some $\bar{M}_{g}>0$,

(ii) $\left\|g\left(t, y, z_{1}\right)-g\left(t, y, z_{2}\right)\right\|_{B L\left(\mathbb{R}^{N}, L^{2}(\mathscr{D})\right)} \leq M_{g}\left|z_{1}-z_{2}\right|$, for all $0 \leq t \leq T, y \in \mathscr{D}$, $z_{1}, z_{2} \in \mathbb{R}$, and some $M_{g}>0$.

(A13) $\xi$ is an $\mathscr{F}_{0}$-measurable random variable independent of $\beta$ with almost surely continuous paths.

We have the following theorem.

THeOREM 6.2. If (A9)-(A13) are satisfied and $4 M_{S}^{2}\left(M_{f_{1}}^{2}+M_{f_{3}}^{2} M_{a}^{2} L_{f_{3}}^{2}\right)<1$, then (6.1) has a unique mild solution $x \in C\left([-r, T] ; L^{2}\left(\Omega, L^{2}(\mathscr{D})\right)\right)$ with probability law $\{\mu(t, \cdot): 0 \leq t \leq T\}$. Proof. Let $H=L^{2}(\mathscr{D})$ and $K=\mathbb{R}^{N}$, denote $\partial x / \partial t$ by $x^{\prime}(t)$, and define the operator $A$ by

$$
A x(t, \cdot)=\Delta_{z} x(t, \cdot), \quad x \in H^{2}(\mathscr{D}) \cap H_{0}^{1}(\mathscr{D}) .
$$

It is known that $A$ generates a strongly continuous semigroup $\{S(t)\}$ on $L^{2}(\mathscr{D})$ (see [26]). Define the maps $f_{1}:[0, T] \times C_{r} \times \wp_{\lambda^{2}}(H) \rightarrow H, f_{3}:[0, T] \times C_{r} \times \wp_{\lambda^{2}}(H) \rightarrow B L(K, H)$, and $\phi:[0, T] \times \mathscr{D} \rightarrow \mathbb{R}$, respectively, by

$$
\begin{gathered}
f_{1}\left(t, x_{t}, \mu(t)\right)(z)=F_{1}(t, z, x(t-r, z))+\int_{L^{2}(\mathscr{D})} F_{2}(t, z, y) \mu(t, z)(d y), \\
f_{3}\left(t, x_{t}, \mu(t)\right)(z)=g(t, z, x(t-r, z)) \quad \forall 0 \leq t \leq T, z \in \mathscr{D}, x_{t} \in C_{r}, \\
\phi(t)(z)=\xi(t, z),
\end{gathered}
$$

where $C_{r}=C_{r}\left([-r, 0] ; L^{2}(\mathscr{D})\right)$. Further, identifying $K_{1}(t, s)$ with $a(t, s)$ and taking $f_{2}=$ $K_{1}=0$, we observe that (6.1) can be written in the abstract form (1.1). From above, (A1) and (A4) are satisfied. We show that $f_{1}$ and $f_{3}$ as in (6.3) and (6.4) satisfy (A2) and (A3). To this end, observe that from (A9)(i), we obtain

$$
\begin{aligned}
\left\|F_{1}\left(t, \cdot, x_{t}(\theta, \cdot)\right)\right\|_{L^{2}(\mathscr{D})} & \leq \bar{M}_{F_{2}}\left[\int_{\mathscr{D}}\left[1+\left|x_{t}(\theta, z)\right|\right]^{2} d z\right]^{1 / 2} \\
& \leq 2 \bar{M}_{F_{1}}\left[m(\mathscr{D})+\left\|x_{t}(\theta, \cdot)\right\|_{L^{2}(\mathscr{D})}^{2}\right]^{1 / 2} \\
& \leq 2 \bar{M}_{F_{1}}\left[\sqrt{m(\mathscr{D})}+\left\|x_{t}\right\|_{C_{r}}\right] \\
& \leq M_{F_{1}}^{*}\left[1+\left\|x_{t}\right\|_{C_{r}}\right] \quad \forall 0 \leq t \leq T, x_{t} \in C_{r},
\end{aligned}
$$

where

$$
M_{F_{1}}^{*}= \begin{cases}2 \bar{M}_{F_{1}} \sqrt{m(\mathscr{D})} & \text { if } m(\mathscr{D})>1, \\ 2 \bar{M}_{F_{1}} & \text { if } m(\mathscr{D}) \leq 1\end{cases}
$$


(Here, $m$ denotes Lebesgue measure in $\mathbb{R}^{N}$.) Also, from (A9)(ii), we get

$$
\begin{aligned}
\left\|F_{1}\left(t, \cdot, x_{t}(\theta, \cdot)\right)-F_{1}\left(t, \cdot, y_{t}(\theta, \cdot)\right)\right\|_{L^{2}(\mathscr{D})} \\
\quad \leq M_{F_{1}}\left[\int_{\mathscr{D}}\left|x_{t}(\theta, z)-y_{t}(\theta, z)\right|^{2} d z\right]^{1 / 2} \\
\quad \leq M_{F_{1}}\left[\sup _{-r \leq \theta \leq 0}\left\|x_{t}(\theta, \cdot)-y_{t}(\theta, \cdot)\right\|_{L^{2}(\mathscr{D})}^{2}\right]^{1 / 2} \\
\quad=M_{F_{1}}\left\|x_{t}-y_{t}\right\|_{C_{r}} .
\end{aligned}
$$

Next, using (A10)(i) together with the Hölder inequality yields

$$
\begin{aligned}
\| \int_{L^{2}(\mathscr{D})} & F_{2}(t, \cdot, y) \mu(t, \cdot)(d y) \|_{L^{2}(\mathscr{D})} \\
= & {\left[\int_{\mathscr{D}}\left[\int_{L^{2}(\mathscr{D})} F_{2}(t, z, y) \mu(t, z)(d y)\right]^{2} d z\right]^{1 / 2} } \\
\leq & {\left[\int_{\mathscr{D}} \int_{L^{2}(\mathscr{D})}\left\|F_{2}(t, z, y)\right\|_{L^{2}(\mathscr{D})}^{2} \mu(t, z)(d y) d z\right]^{1 / 2} } \\
\leq & \bar{M}_{F_{2}}\left[\int_{\mathscr{D}}\left(\int_{L^{2}(\mathscr{D})}\left(1+\|y\|_{L^{2}(\mathscr{D})}\right)^{2} \mu(t, z)(d y)\right) d z\right]^{1 / 2} \\
\leq & \bar{M}_{F_{2}} \sqrt{m(\mathscr{D})} \sqrt{\|\mu(t)\|_{\lambda^{2}}}(\mathrm{cf} .(2.2)) \\
\leq & \bar{M}_{F_{2}} \sqrt{m(\mathscr{D})}\left(1+\|\mu(t)\|_{\lambda^{2}}\right) \quad \forall 0 \leq t \leq T, \mu \in \wp_{\lambda^{2}}(H) .
\end{aligned}
$$

Also, invoking (A10)(ii) enables us to see that for all $\mu, \nu \in \wp_{\lambda^{2}}(H)$,

$$
\begin{aligned}
& \left\|\int_{L^{2}(\mathscr{D})} F_{2}(t, \cdot, y) \mu(t, \cdot)(d y)-\int_{L^{2}(\mathscr{D})} F_{2}(t, \cdot, y) \nu(t, \cdot)(d y)\right\|_{L^{2}(\mathscr{D})} \\
& \quad=\left\|\int_{L^{2}(\mathscr{D})} F_{2}(t, \cdot, y)(\mu(t, \cdot)-v(t, \cdot))(d y)\right\|_{L^{2}(\mathscr{D})} \\
& \quad \leq\|\rho(\mu(t), \nu(t))\|_{L^{2}(\mathscr{D})} \quad(\mathrm{cf} .(2.2)) \\
& \quad=\sqrt{m(\mathscr{D})} \rho(\mu(t), \nu(t)) \quad \forall 0 \leq t \leq T .
\end{aligned}
$$

Combining (6.6) and (6.9), we see that $f_{1}$ satisfies (A2)(i) with $\bar{M}_{f_{1}}=2 \cdot \max \left\{\bar{M}_{F_{2}} \sqrt{m(\mathscr{D})}\right.$, $M_{F_{1}}^{*}$, and combining (6.8) and (6.10) shows that $f_{1}$ satisfies (A2)(ii) with $M_{f_{1}}=\max \left\{M_{F_{1}}\right.$, $\sqrt{m(\mathscr{D})}\}$. It is easy to see that $f_{3}$ satisfies (A3) with $M_{f_{3}}=M_{g}$ and $\bar{M}_{f_{3}}=\bar{M}_{g}$. Thus, we can invoke Theorem 3.2 to conclude that (6.1) has a unique mild solution $x \in C([-r, T]$; $\left.L^{2}\left(\Omega, L^{2}(\mathscr{D})\right)\right)$ with probability law $\{\mu(t, \cdot): 0 \leq t \leq T\}$. 
Example 6.3. Consider the following initial-boundary value problem of Sobolev type:

$$
\begin{gathered}
\frac{\partial}{\partial t}\left(x(t, z)-x_{z z}(t, z)\right)-x_{z z}(t, z) \\
=F_{1}(t, z, x(t-r, z))+\int_{L^{2}(0, \pi)} F_{2}(t, z, y) \mu(t, z)(d y) \\
+\int_{0}^{t} a(t, s) g(s, z, x(s-r, z)) d W(s), \quad 0 \leq z \leq \pi, 0 \leq t \leq T, \\
x(t, 0)=x(t, \pi)=0, \quad 0 \leq t \leq T, \\
x(t, z)=\phi(t, z), \quad 0 \leq z \leq \pi,-r \leq t \leq 0,
\end{gathered}
$$

where $x:[0, T] \times[0, \pi] \rightarrow \mathbb{R}$ is the state process, $F_{1}:[0, T] \times[0, \pi] \times \mathbb{R} \rightarrow \mathbb{R}, F_{2}:[0, T] \times$ $[0, \pi] \times L^{2}(0, \pi) \rightarrow L^{2}(0, \pi), a: \Delta \rightarrow \mathbb{R}, g:[0, T] \times[0, \pi] \times \mathbb{R} \rightarrow B L\left(\mathbb{R}, L^{2}(0, \pi)\right)$, and $\phi:$ $[0, T] \times[0, \pi] \rightarrow \mathbb{R}$ are mappings satisfying (A9)-(A13) (in the appropriate spaces), $\mu(t, \cdot)$ $\in \wp_{\lambda^{2}}\left(L^{2}(0, \pi)\right)$ is the probability law of $x(t, \cdot)$, and $W$ is a standard $L^{2}(0, \pi)$-valued Wiener process. We have the following theorem.

THEOREM 6.4. Under the above assumptions, (6.11) has a unique mild solution $x \in$ $C\left([-r, T] ; L^{2}\left(\Omega, L^{2}(0, \pi)\right)\right)$, provided that $4\left(M_{f_{1}}^{2}+M_{f_{3}}^{2} M_{a}^{2} L_{f_{3}}^{2}\right)<1$.

Proof. Let $H=L^{2}(0, \pi), K=\mathbb{R}$, and define the operators $A: D(A) \subset H \rightarrow H$ and $B$ : $D(B) \subset H \rightarrow H$, respectively, by

$$
A x(t, \cdot)=-x_{z z}(t, \cdot), \quad B x(t, \cdot)=x(t, \cdot)-x_{z z}(t, \cdot),
$$

with domains

$$
\begin{aligned}
D(A)= & D(B) \\
= & \left\{x \in L^{2}(0, \pi): x, x_{z}\right. \text { are absolutely continuous, } \\
& \left.x_{z z} \in L^{2}(0, \pi), x(0)=x(\pi)=0\right\} .
\end{aligned}
$$

Define $\phi, K_{1}, K_{2}$, and $f_{i}(i=1,2,3)$ as in Example 6.1 (with $L^{2}(0, \pi)$ in place of $L^{2}(\mathscr{D})$ ). Then, (6.11) can be written in the abstract form

$$
\begin{gathered}
(B x(t))^{\prime}+A x(t)=f_{1}\left(t, x_{t}, \mu(t)\right)+\int_{0}^{t} a(t, s) f_{3}\left(s, x_{s}, \mu(s)\right) d W(s), \quad 0 \leq t \leq T, \\
x(t)=\phi(t), \quad-r \leq t \leq 0 .
\end{gathered}
$$

Upon making the substitution $v(t)=B x(t)$ in (6.14), we arrive at the equivalent problem

$$
\begin{aligned}
v^{\prime}(t)+A B^{-1} v(t)= & f_{1}\left(t, B^{-1} v_{t}, \mu(t)\right) \\
& +\int_{0}^{t} a(t, s) f_{3}\left(s, B^{-1} v_{s}, \mu(s)\right) d W(s), \quad 0 \leq t \leq T, \\
v(t)= & B \phi(t), \quad-r \leq t \leq 0 .
\end{aligned}
$$


It is known that $B$ is a bijective operator possessing a continuous inverse and that $-A B^{-1}$ is a bounded linear operator on $L^{2}(0, \pi)$ which generates a strongly continuous semigroup $\{T(t)\}$ on $L^{2}(0, \pi)$ satisfying (A1) with $M_{T}=\alpha=1$ (see [26]). Further, $f_{1}$ and $f_{3}$ are shown to satisfy (A2) and (A3) as in Example 6.1. Consequently, we can invoke Theorem 3.2 (assuming that $4\left(M_{f_{1}}^{2}+M_{f_{3}}^{2} M_{a}^{2} L_{f_{3}}^{2}\right)<1$ ) to conclude that $(6.15)$ has a unique mild solution $v \in C\left([-r, T] ; L^{2}\left(\Omega, L^{2}(0, \pi)\right)\right)$. Consequently, $x=B^{-1} v$ is the corresponding mild solution of (6.14) and hence, of (6.11).

Remark 6.5. This example provides a generalization of the work in $[2,7,22,27,28]$ to the stochastic setting. Such initial-boundary value problems arise naturally in the mathematical modeling of various physical phenomena (e.g., thermodynamics [8], shear in second-order fluids [15,33], fluid flow through fissured rocks [3], and consolidation of clay [32]).

\section{References}

[1] N. U. Ahmed and X. Ding, A semilinear McKean-Vlasov stochastic evolution equation in Hilbert space, Stochastic Process. Appl. 60 (1995), no. 1, 65-85.

[2] K. Balachandran and R. Sakthivel, Controllability of Sobolev-type semilinear integrodifferential systems in Banach spaces, Appl. Math. Lett. 12 (1999), no. 7, 63-71.

[3] G. I. Barenblatt, I. P. Zheltov, and I. N. Kochina, Basic concepts in the theory of seepage of homogeneous liquids in fissured rocks, J. Appl. Math. Mech. 24 (1960), 1286-1303.

[4] H. Bergström, Weak Convergence of Measures, Probability and Mathematical Statistics, Academic Press, New York, 1982.

[5] A. T. Bharucha-Reid, Random Integral Equations, Mathematics in Science and Engineering, vol. 96, Academic Press, New York, 1972.

[6] P. Billingsley, Weak Convergence of Measures: Applications in Probability, Society for Industrial and Applied Mathematics, Pennsylvania, 1971.

[7] H. Brill, A semilinear Sobolev evolution equation in a Banach space, J. Differential Equations 24 (1977), no. 3, 412-425.

[8] P. J. Chen and M. E. Gurtin, On a theory of heat conduction involving two temperatures, Z. Angew. Math. Phys. 19 (1968), 614-627.

[9] M. G. Crandall, S.-O. Londen, and J. A. Nohel, An abstract nonlinear Volterra integrodifferential equation, J. Math. Anal. Appl. 64 (1978), no. 3, 701-735.

[10] G. Da Prato and J. Zabczyk, Stochastic Equations in Infinite Dimensions, Encyclopedia of Mathematics and Its Applications, vol. 44, Cambridge University Press, Cambridge, 1992.

[11] D. A. Dawson, Critical dynamics and fluctuations for a mean-field model of cooperative behavior, J. Statist. Phys. 31 (1983), no. 1, 29-85.

[12] D. A. Dawson and J. Gärtner, Large deviations from the McKean-Vlasov limit for weakly interacting diffusions, Stochastics 20 (1987), no. 4, 247-308.

[13] T. E. Govindan, Autonomous semilinear stochastic Volterra integro-differential equations in Hilbert spaces, Dynam. Systems Appl. 3 (1994), no. 1, 51-74.

[14] W. Grecksch and C. Tudor, Stochastic Evolution Equations. A Hilbert Space Approach, Mathematical Research, vol. 85, Akademie-Verlag, Berlin, 1995.

[15] R. R. Huilgol, A second order fluid of the differential type, Internat. J. Non-Linear Mech. 3 (1968), no. 4, 471-482.

[16] A. Ichikawa, Stability of semilinear stochastic evolution equations, J. Math. Anal. Appl. 90 (1982), no. $1,12-44$. 
[17] D. Kannan, Random integrodifferential equations, Probabilistic Analysis and Related Topics, Vol. 1 (A. T. Bharucha-Reid, ed.), Academic Press, New York, 1978, pp. 87-167.

[18] D. Kannan and A. T. Bharucha-Reid, On a stochastic integro-differential evolution equation of Volterra type, J. Integral Equations 10 (1985), no. 1-3, suppl., 351-379.

[19] D. N. Keck and M. A. McKibben, On a McKean-Vlasov stochastic integro-differential evolution equation of Sobolev-type, Stochastic Anal. Appl. 21 (2003), no. 5, 1115-1139.

[20] K. Knopp, Theory and Application of Infinite Series, Dover Publications, New York, 1990.

[21] H. Kunita, Stochastic Flows and Stochastic Differential Equations, Cambridge Studies in Advanced Mathematics, vol. 24, Cambridge University Press, Cambridge, 1990.

[22] J. H. Lightbourne III and S. M. Rankin III, A partial functional-differential equation of Sobolev type, J. Math. Anal. Appl. 93 (1983), no. 2, 328-337.

[23] S.-O. Londen and J. A. Nohel, Nonlinear Volterra integro-differential equation occurring in heat flow, J. Integral Equations 6 (1984), no. 1, 11-50.

[24] M. Nagasawa and H. Tanaka, Diffusion with interactions and collisions between coloured particles and the propagation of chaos, Probab. Theory Related Fields 74 (1987), no. 2, 161-198.

[25] B. G. Pachpatte, Inequalities for Differential and Integral Equations, Mathematics in Science and Engineering, vol. 197, Academic Press, California, 1998.

[26] A. Pazy, Semigroups of Linear Operators and Applications to Partial Differential Equations, Applied Mathematical Sciences, vol. 44, Springer-Verlag, New York, 1983.

[27] R. E. Showalter, A nonlinear parabolic-Sobolev equation, J. Math. Anal. Appl. 50 (1975), 183190.

[28]_- Nonlinear degenerate evolution equations and partial differential equations of mixed type, SIAM J. Math. Anal. 6 (1975), 25-42.

[29] H. Tanabe, Equations of Evolution, Monographs and Studies in Mathematics, vol. 6, Pitman, Massachusetts, 1979.

[30] T. Taniguchi, Almost sure exponential stability for stochastic partial functional-differential equations, Stochastic Anal. Appl. 16 (1998), no. 5, 965-975.

[31] T. Taniguchi, K. Liu, and A. Truman, Existence, uniqueness, and asymptotic behavior of mild solutions to stochastic functional differential equations in Hilbert spaces, J. Differential Equations 181 (2002), no. 1, 72-91.

[32] D. Taylor, Research on Consolidation of Clays, MIT Press, Cambridge, 1952.

[33] T. W. Ting, Certain non-steady flows of second-order fluids, Arch. Rational Mech. Anal. 14 (1963), 1-26.

[34] J. Wu, Theory and Applications of Partial Functional-Differential Equations, Applied Mathematical Sciences, vol. 119, Springer-Verlag, New York, 1996.

David N. Keck: Department of Mathematics, Ohio University, Athens, OH 45701, USA

E-mail address: dkeck@bing.math.ohiou.edu

Mark A. McKibben: Mathematics and Computer Science Department, Goucher College, Baltimore, MD 21204, USA

E-mail address: mmckibben@goucher.edu 


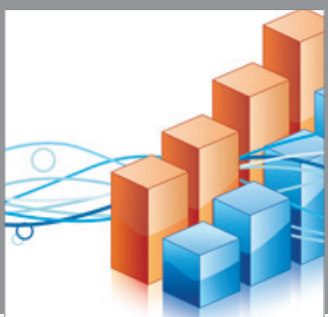

Advances in

Operations Research

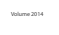

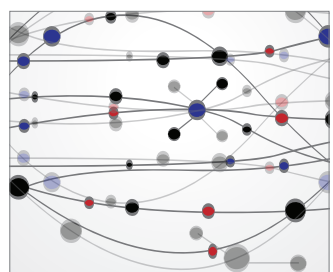

\section{The Scientific} World Journal
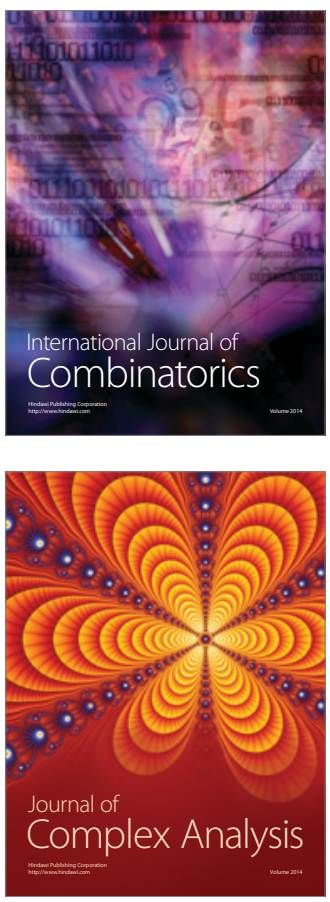

International Journal of

Mathematics and

Mathematical

Sciences
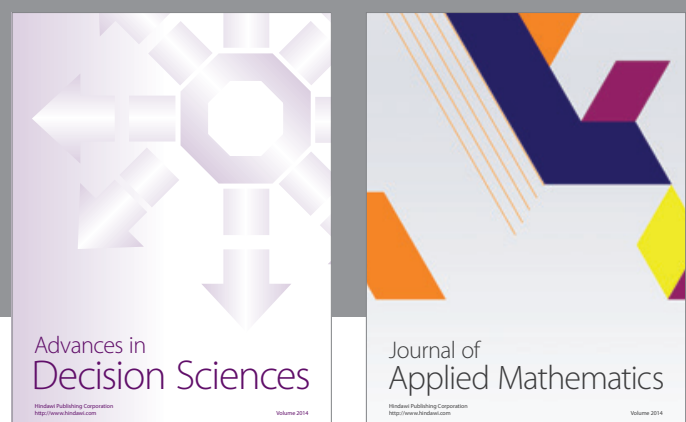

Journal of

Applied Mathematics
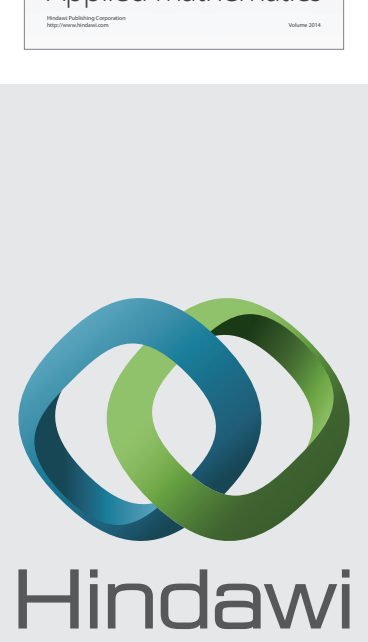

Submit your manuscripts at http://www.hindawi.com
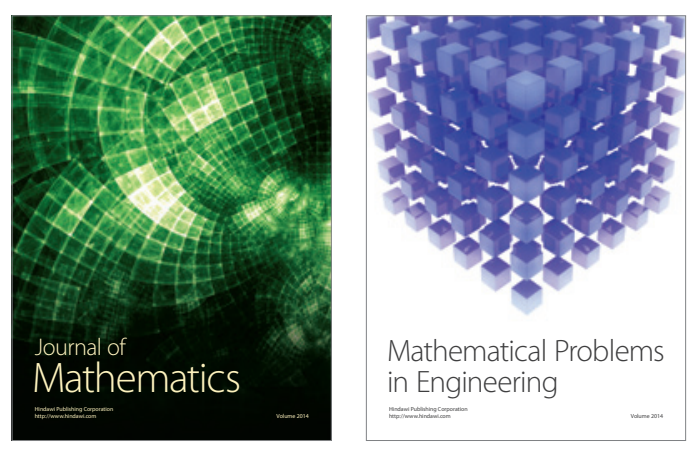

Mathematical Problems in Engineering
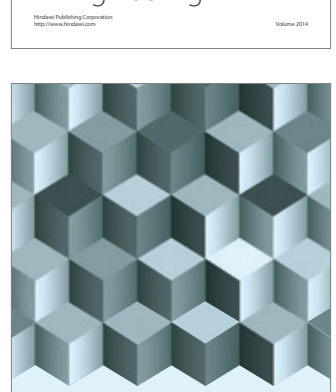

Journal of

Function Spaces
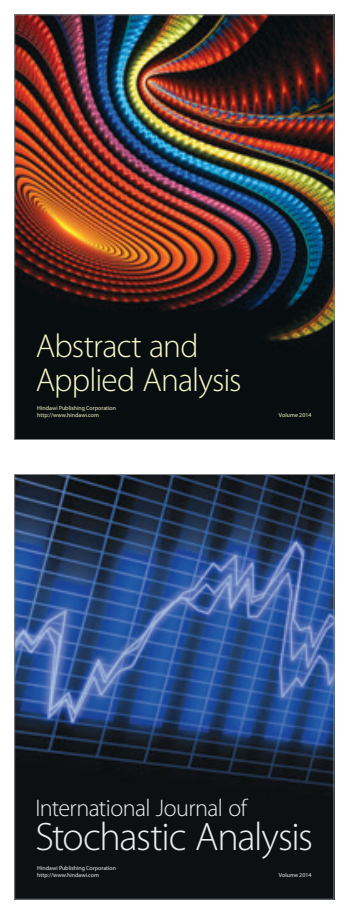

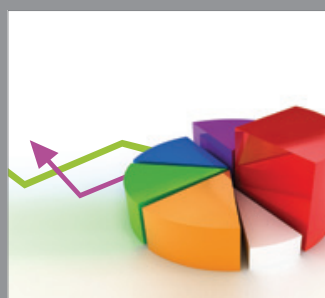

ournal of

Probability and Statistics

Promensencen
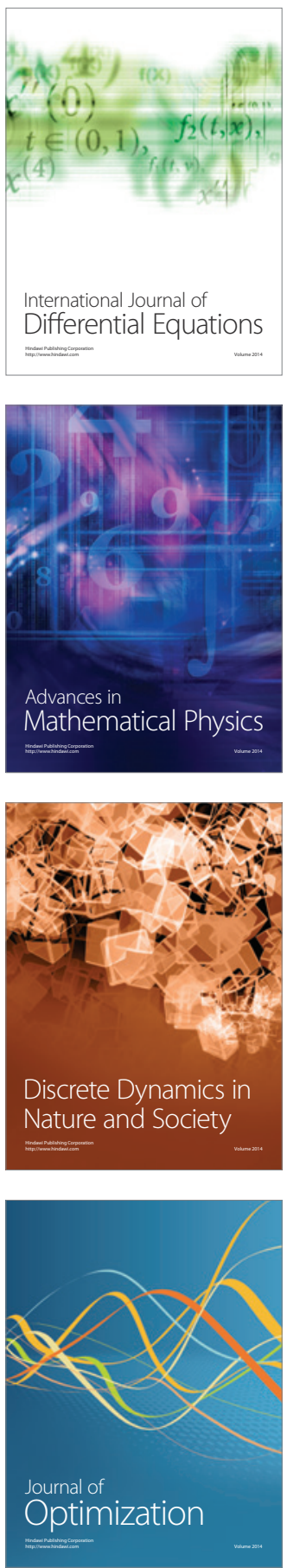